\title{
Recherches expérimentales sur le chant diphonique
}

Hugo Zemp et Trân Quang Hai

\section{OpenEdition}

Édition électronique

URL : http://journals.openedition.org/ethnomusicologie/1572

ISSN : 2235-7688

\section{Éditeur}

ADEM - Ateliers d'ethnomusicologie

\section{Édition imprimée}

Date de publication : 1 octobre 1991

Pagination : 27-68

ISBN : 978-2-8257-0437-8

ISSN : $1662-372 X$

\section{Référence électronique}

Hugo Zemp et Trân Quang Hai, «Recherches expérimentales sur le chant diphonique », Cahiers

d'ethnomusicologie [En ligne], 4 | 1991, mis en ligne le 01 janvier 2012, consulté le 10 décembre 2020 URL : http://journals.openedition.org/ethnomusicologie/1572 


\title{
RECHERCHES EXPÉRIMENTALES SUR LE CHANT DIPHONIQUE
}

\author{
Hugo Zemp et Trân Quang Hai
}

La dénomination «chant diphonique» désigne une technique vocale singulière selon laquelle une seule personne chante à deux voix: un bourdon constitué par le son fondamental, et une mélodie superposée formée par des harmoniques.

Cet article est issu de deux approches complémentaires: une recherche pragmatique par l'apprentissage et l'exercice du chant diphonique que Trân Quang Hai mène depuis 1971, et une recherche de visualisation conduite sur le plan physiologique et acoustique pour la préparation et la production du film Le chant des harmoniques, réalisé par Hugo Zemp en 1988-89. Dans ce film, Trân Quang Hai est l'acteur principal, tour à tour chanteur et ethnomusicologue: enseignant le chant diphonique lors d'un atelier, interviewant des chanteurs mongols, se prêtant à la radiocinématographie avec traitement informatique de l'image, et chantant dans le microphone du spectrographe pour analyser ensuite sa propre technique vocale ${ }^{2}$. Les images spectrographiques que nous avons découvertes pratiquement en même temps que nous les filmions - le dernier modèle de Sona-Graph permettant l'analyse du spectre sonore en temps réel et son synchrone était arrivé au Département d'ethnomusicologie du Musée de l'Homme quelques jours avant le tournage - nous ont encouragés à poursuivre ces investigations et à les conduire dans une direction que nous n'aurions probablement pas envisagée sans la réalisation $\mathrm{du}_{\text {film }}^{3}$.

Ces recherches ont été effectuées dans le cadre de l'Unité Propre de Recherche no 165 du CNRS, au Département d'ethnomusicologie du Musée de l'Homme. Avant d'envoyer le manuscrit final à l'éditeur, nous avons pu présenter au séminaire de l'UPR un résumé de notre travail. Les questions et remarques nous ont aidé à corriger et préciser notre analyse. Gilles Léothaud et Gilbert Rouget nous ont alors fait l'amitié de relire le manuscrit et de nous faire part de leurs suggestions pour l'améliorer. Nous voudrions exprimer à tous notre gratitude.

2 Les détails de la réalisation de ce film - dont la première a eu lieu le 27 juillet 1989 lors du Congrès de l'International Council for Traditional Music à Schladming (Autriche) - sont décrits ailleurs (cf. Zemp 1989). Un livret, devant accompagner l'édition sous forme de vidéocassette, est actuellement en préparation.

Recherche menée en étroite collaboration par les deux co-auteurs qui chacun ont apporté - en plus de l'évaluation en commun de chaque étape du travail - des contributions spécifiques. Les sonagrammes, ainsi que les analyses détaillées ayant servi à rédiger les légendes des figures, sont de Trân Quang Hai qui, en plus, est en même temps «informateur privilégié» et chanteur de 26 enregistrements reproduits sur sonagrammes. La conception de la recherche, la mise en forme et la rédaction de l'article sont de Hugo Zemp. 
L'utilisation des outils spectrographiques pour l'analyse des chants diphoniques n'est pas nouvelle: Leipp (1971), Hamayon (1973), Walcott (1974), BorelMaisonny et Castellengo (1976), Trân Quang Hai et Guillou (1980), Gunji (1980), Harvilahti (1983), Harvilahti et Kaskinen (1983), Desjacques (1988), Léothaud (1989). Il n'est pas question, dans le cadre de cet article, d'évaluer ces travaux, d'en résumer les résultats ou d'en faire l'historique. Dans l'étude la plus récente, G. Léothaud (1989: 20-21) ${ }^{4}$ résume excellemment ce qu'il appelle la «genèse acoustique du chant diphonique»:

L'appareil phonatoire, comme tout instrument de musique, se compose d'un système excitateur, ici le larynx, et d'un corps vibrant chargé de transformer l'énergie reçue en rayonnement acoustique, le conduit pharyngo-buccal.

Le larynx délivre un spectre harmonique, le son laryngé primaire, déterminé en fréquence, d'allure homogène, c'est-à-dire dénué de formants notables - donc de couleur vocalique - et dont la richesse en harmoniques varie essentiellement en fonction de la structure vibratoire des cordes vocales [...].

Cette fourniture primaire traverse les cavités pharyngo-buccales, y subissant d'importantes distorsions: le pharynx et la bouche se comportent donc comme des résonateurs de Helmholtz, et cela pour toutes les fréquences dont la longueur d'onde est supérieure à la plus grande dimension de ces cavités. [...]

Les paramètres déterminant la fréquence propre des cavités phonatoires peuvent varier dans des proportions considérables grâce au système articulateur, notamment par la mobilité de la mâchoire, l'ouverture de la bouche et la position de la langue. Celle-ci, surtout, peut diviser la cavité buccale en deux résonateurs de plus petit volume, donc de fréquence propre plus élevée. En d'autres termes, les cavités buccales peuvent continuer à se comporter en résonateurs de Helmholtz même pour des harmoniques très aigus du spectre laryngé, ceux dont la longueur d'onde est petite, et en tout cas inférieure à la longueur du conduit pharyngo-buccal.

L'émission diphonique consiste pour le chanteur à émettre un spectre riche en harmoniques, puis à accorder très finement une cavité phonatoire sur l'un des composants de ce spectre, dont l'amplitude augmente ainsi fortement par résonance; par déplacement de la langue, le volume buccal peut varier, donc la fréquence propre, et sélectionner de cette façon différents harmoniques.

Il propose une grille d'analyse, axée sur quatre niveaux et douze critères pertinents. $1^{\circ}$ Caractéristiques du spectre vocal; $2^{\circ}$ Nature du formant diphonique; $3^{\circ}$ Caractéristiques de la mélodie d'harmoniques; $4^{\circ}$ Champ de liberté de la

Paru dans une nouvelle publication: le Dossier $n^{\circ} 1$ de l'Institut de la Voix, Limoges. Outre deux brefs rapports relatant des examens cliniques et paracliniques de l'appareil phonatoire et de l'émission diphonique de Trân Quang Hai, examens effectués l'un par un médecin O.R.L. (Sauvage 1989) et l'autre par un phoniatre (Pailler 1989), et l'extrait d'un exposé sur la réalisation du chant diphonique (Trân Quang Hai 1989), ce dossier contient également la bibliographie et la discographie les plus complètes à ce jour concernant le chant diphonique. 
fluctuation diphonique. L'application de cette grille permet d'approfondir et de systématiser l'analyse spectrale du chant diphonique qui peut maintenant s'appuyer sur de nombreux nouveaux documents sonores publiés récemment sur des disques, s'ajoutant aux anciens bien connus. Cependant, tel n'est pas notre but.

Nous nous proposons d'examiner comment les différents styles ou variantes stylistiques du chant diphonique - appelé chez les Mongols khöömii («pharynx, gorge») et chez les Tuva de l'URSS khomei (du terme mongol) - sont produits sur le plan physiologique. Dans ce domaine, les descriptions sont rares et peu détaillées, alors qu'on connaît depuis de nombreuses années les noms vernaculaires désignant ces styles chez les Tuva dont Aksenov (1973: 12) pense qu'ils forment le centre de la culture turco-mongole du chant diphonique, puisqu'ils ne pratiquent pas seulement une mais quatre variantes stylistiques (kargiraa, borbannadir, sigit, ezengileer; un cinquième nom, khomei qui est en même temps le nom générique du chant diphonique, remplaçant dans certains lieux le terme borbannadir). Implicitement, les peuples voisins qu'il cite - Mongols, Oirats, Kharkass, Gorno-Altaïs et Bashkirs - n'en connaîtraient qu'un seul style. En tout cas, pour les Mongols et pour les Altaï de l'URSS, montagnards habitant la chaîne du même nom, cela n'est pas exact. Les derniers utilisent trois styles nommés sur la notice d'un disque sibiski, karkira, kiomioi (Petrov et Tikhonurov). Le chanteur diphonique le plus connu en Mongolie et à l'étranger, D. Sundui, a énuméré cinq styles lors du festival Musical Voices of Asia au Japon: xarkiraa xöömij (xöömij narratif), xamrijn хӧömij (xöömij de nez), bagalzuurijn xöömij (xöömij de gorge), tseedznii xöömij (xöömij de poitrine), kevliin xöömij (xöömij de ventre), les deux derniers n'étant généralement pas différenciés (Emmert et Minegishi 1980: 48). Dans l'interview du film Le chant des harmoniques, T. Ganbold indique les même cinq noms. Il présente brièvement les quatres premiers styles, en ajoutant qu'il ne sait pas faire le «khöömii de ventre», le distinguant par là du «khöömii de poitrine». Mais il n'explique pas comment il produit ces différents styles. Il est vrai que l'interview avait dû être réalisé en très peu de temps, en économisant de la pellicule, et avec l'aide d'un traducteur, fonctionnaire du Ministère des Affaires étrangères de Mongolie, probablement peu familier avec les subtilités du chant. T. Ganbold et G. Iavgaan avaient également dirigé plusieurs ateliers à la Maison des cultures du monde à Paris; cette fois-ci la traduction était assurée par un ethnomusicologue, Alain Desjacques, mais les deux chanteurs n'en étaient pas plus explicites. Quant à D. Sundui, à qui un musicologue japonais demandait comment faire pour apprendre le chant diphonique, il répondait simplement qu'il fallait savoir tenir son souffle aussi longtemps que possible, l'utiliser efficacement, puis écouter des enregistrements sonores et essayer (Emmert et Minegushi 1980: 49).

Malgré le fait que son pays d'origine (le Vietnam) et son pays d'accueil (la France) ne connaissent pas traditionnellement le chant diphonique - ou

La translittération change selon les auteurs xöömij, khöömii, chöömij, ho-mi. 
peut-être grâce à cela - Trân Quang Hai réussit à reproduire différents styles ou variantes stylistiques, ou du moins à s'en approcher. Ayant appris sans recevoir d'instructions ou de conseils de chanteurs chevronnés, et sans pouvoir s'appuyer sur des descriptions publiées, il a été obligé de procéder par tâtonnement. Cette recherche empirique, mais néanmoins systématique, lui a permis de prendre conscience de ce qui se passe au niveau de la cavité buccale. Conduire depuis de nombreuses années des ateliers d'introduction au chant diphonique l'a amené à savoir l'expliciter.

L'originalité des nouvelles recherches présentées ici consiste en 3 points:

1) Trân Quang Hai essaie d'imiter le mieux possible des chants reproduits sur les enregistrements sonores dont nous disposons. Pour cela, il s'appuie à la fois sur la perception auditive et visuelle, en essayant d'obtenir, sur le moniteur du Sona-Graph, des tracés de spectres semblables à ceux de chanteurs originaires de Mongolie, de Sibérie, du Rajasthan et d'Afrique du Sud.

2) Il décrit subjectivement ce qu'il fait et ressent sur le plan physiologique, quand il obtient ces tracés.

3) Afin de mieux comprendre le mécanisme des différents styles et d'en explorer toutes les possibilités - même si elles ne sont pas exploitées dans les chants diphoniques traditionnels -, il effectue des expériences que nul n'a probablement jamais tentées.

Cette recherche ne pouvait être effectuée avec des spectrographes de facture ancienne utilisés jusqu'en 1989 par les auteurs mentionnés plus haut. Il fallait pour cela un appareil capable de restituer le spectre sonore en temps réel et son synchrone, le DSP Sona-Graph Model 5500 que notre équipe de recherche acquit en décembre 1988. Si l'on change, en chantant, les paramètres de l'émission vocale, on voit immédiatement se modifier le tracé des harmoniques. Grâce au feed-back du nouveau tracé, l'émission vocale peut de nouveau être modifiée. Ainsi, la recherche est proprement expérimentale.

Dans la première étude sur l'acoustique du chant diphonique, E. Leipp reproduit un schéma théorique de l'appareil phonatoire, figurant cinq cavités principales comme résonateurs: $1^{\circ}$ la cavité pharyngienne; $2^{\circ}$ la cavité buccale postérieure $; 3^{\circ}$ la cavité buccale antérieure, la pointe de la langue dirigée vers le palais séparant les cavités 2 et $3 ; 4^{\circ}$ la cavité située entre les dents et les lèvres; $5^{\circ}$ la cavité nasale (Leipp 1971). Le rôle exact de ces différentes cavités semble difficile à définir.

Grâce à son expérience pragmatique de chanteur et de pédagogue, Trân Quang Hai a été amené à distinguer deux techniques de base utilisant essentiellement une cavité buccale ou deux cavités buccales (Trân et Guillou 1980:171), les deux techniques pouvant être plus ou moins nasalisées. Dans la technique à une cavité, la pointe de la langue reste en bas, comme lorsqu'on prononce des voyelles. Trân Quang Hai a trouvé cette technique parfaite pour mieux faire sentir aux débutants la modification du volume buccal avec la prononciation 
des voyelles. Il dit aux stagiaires qu'il faut «laisser la langue en position de repos» (cf. le film Le chant des harmoniques). Les images radiologiques du film montrent cependant que l'arrière de la langue se lève pendant la prononciation successive des voyelles o, ว, a (ceci n'est pas lié au chant diphonique). Le radiologiste F. Besse parle de «l'ascension de la langue». La métaphore du «repos de la langue » reste pourtant valable dans le sens où la pointe de la langue reste en bas. L'image radiologique montre que dans cette technique, il y a un contact entre le voile du palais et la partie postérieure de la langue, séparant la cavité buccale de la zone pharyngienne.

Dans la technique à deux cavités, la pointe de la langue est appliquée contre la voûte du palais, divisant ainsi le volume buccal en une cavité antérieure et une cavité postérieure. Ici, il n'y a pas de contact entre l'arrière de la langue et le voile du palais; la cavité buccale postérieure et la cavité pharyngienne étant reliées par un large passage. La sélection des différents harmoniques pour créer une mélodie peut se faire de deux manières: a) la pointe de la langue se déplace de l'arrière à l'avant, l'harmonique le plus aigu étant obtenu dans la position le plus en avant; la cavité buccale antérieure est alors réduite au maximum (cf. les images radiologiques du film Le chant des harmoniques); b) la pointe de la langue reste collée au palais sans se déplacer, les harmoniques étant sélectionnés en fonction de l'ouverture plus ou moins grande des lèvres: de l'ouverture la plus petite quand on prononce la voyelle o (harmonique grave) jusqu'à l'ouverture la plus grande quand on prononce la voyelle $\underline{\mathrm{i}}$ (harmonique aigu). Cette deuxième manière ne semble pas être utilisée par les chanteurs mongols que nous avons pu observer, et Trân Quang Hai ne l'emploie que pour son intérêt pédagogique (comparaison avec la technique à une cavité) lors de ses ateliers d'initiation.

Afin d'explorer toutes les possibilités des deux techniques principales, Hai a chanté des échelles d'harmoniques à partir de différentes hauteurs du fondamental. Pour la technique à une cavité, on s'aperçoit sur la figure 1 que les harmoniques utilisables pour créer une mélodie ne dépassent que de peu la limite supérieure de $1000 \mathrm{~Hz}$, quel que soit le fondamental. Mais plus le fondamental est grave, plus les harmoniques sont nombreux. Ainsi, pour le fondamental le plus grave ( $90 \mathrm{~Hz}$, approximativement un fa1) de la fig. 1, les harmoniques exploitables sont $\mathrm{H} 4(360 \mathrm{~Hz}), 5,6,7,8,9,10,11,12(1080 \mathrm{~Hz})$, ce qui donne l'échelle (transposée) do, mi, sol, sib-, do, re, mi, fa\#-, sol. Pour le fondamental le plus aigu $(180 \mathrm{~Hz})$ de la fig. 1, seuls les harmoniques 3, 4, 5 et 6 sont exploitables, et l'échelle résultante, sol, do, mi, sol, est beaucoup plus pauvre en possibilités mélodiques.

Avec la technique à deux cavités (fig. 2), et le fondamental le plus grave (110 $\mathrm{Hz}=\mathrm{La} 1)$, Trân Quang Hai arrive à faire ressortir les harmoniques entre H6 $(660 \mathrm{~Hz})$ et H20 $(2200 \mathrm{~Hz})$. Pour créer une mélodie dans la zone la plus aiguë, il faut sélectionner des harmoniques pairs ou impairs (cf. plus loin fig. 11 et 12), puisque les harmoniques sont trop rapprochés pour une échelle musicale. L'émission du fondamental le plus aigu $(220 \mathrm{~Hz})$ de la fig. 2 permet de sélectionner de H4 $(880 \mathrm{~Hz})$ à H10 $(2200 \mathrm{~Hz})$. 


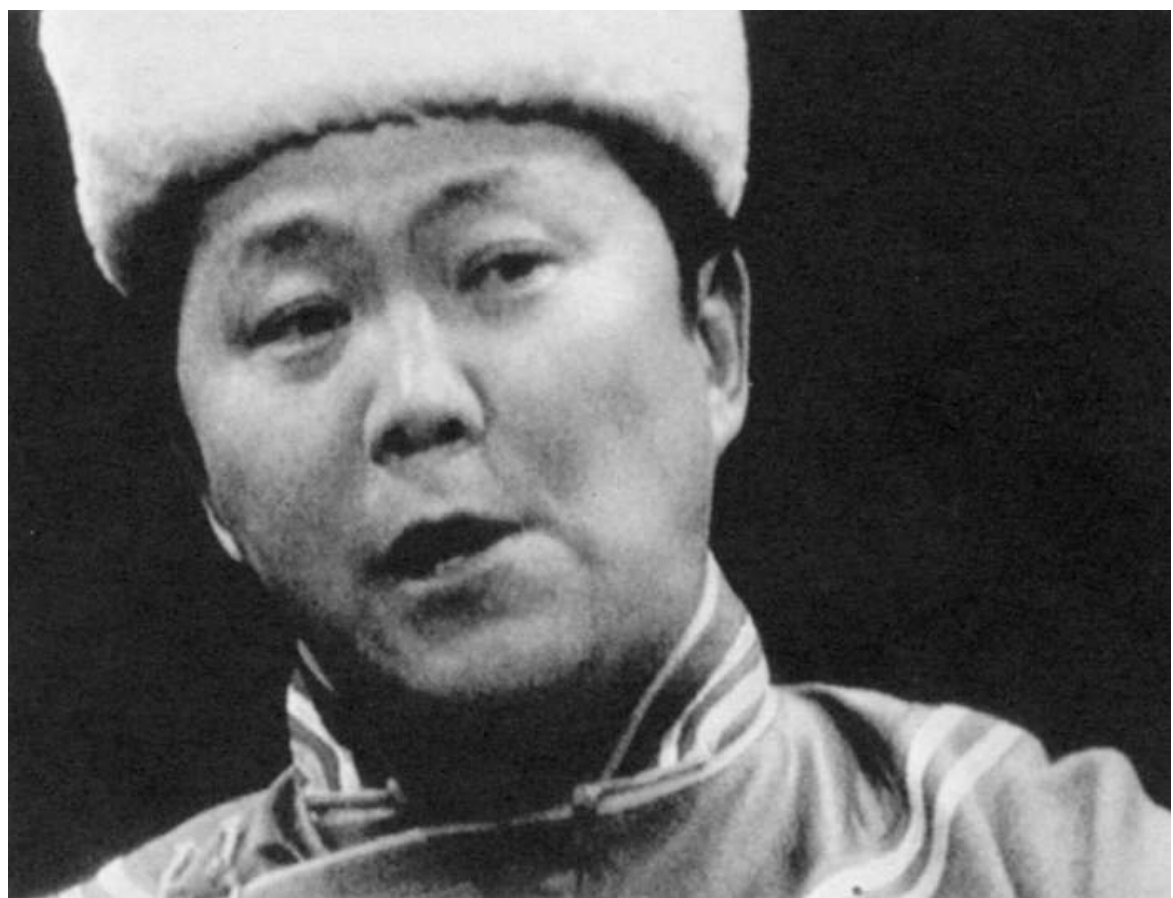

G. YAVGAAN. Photogramme extrait du film «Le chant des harmoniques» de H. Zemp.

Un rapide coup d'œil permet de constater qu'en fait, les harmoniques obtenus par la technique à une cavité se situent essentiellement dans une zone jusqu'à $1 \mathrm{KHz}$, alors que les harmoniques obtenus par la technique à deux cavités sont placés surtout dans la zone de 1 à $2 \mathrm{KHz}$.

Dans la tradition, les femmes mongoles et tuva ne pratiquaient pas le chant diphonique. Selon le chanteur D. Sundui, cette pratique nécessiterait trop de force, mais il n'y aurait pas d'interdit à ce sujet chez les Mongols (Emmert et Minegushi 1980: 48). Chez les Tuva de l'Union soviétique, le chant diphonique serait presque exclusivement réservé aux hommes; un tabou basé sur la croyance qu'il causerait l'infertilité à la femme qui le pratiquerait serait progressivement abandonné, et quelques jeunes filles l'apprendraient maintenant (Alekseev, Kirgiz et Levin 1990). Ces auteurs disent encore que «les femmes sont capables de produire les même sons, bien qu'à des hauteurs plus élevés », ce qui n'est que partiellement vrai. C'est vrai si on parle «des sons » du bourdon qui sont plus élevés pour une voix de femme que pour une voix d'homme, mais c'est faux en ce qui concerne la mélodie d'harmoniques qui ne peut monter plus haut que chez les hommes. On peut déjà le déduire en examinant les fig. 1 et 2 où la limite supérieure des harmoniques obtenus à partir des fondamentaux 


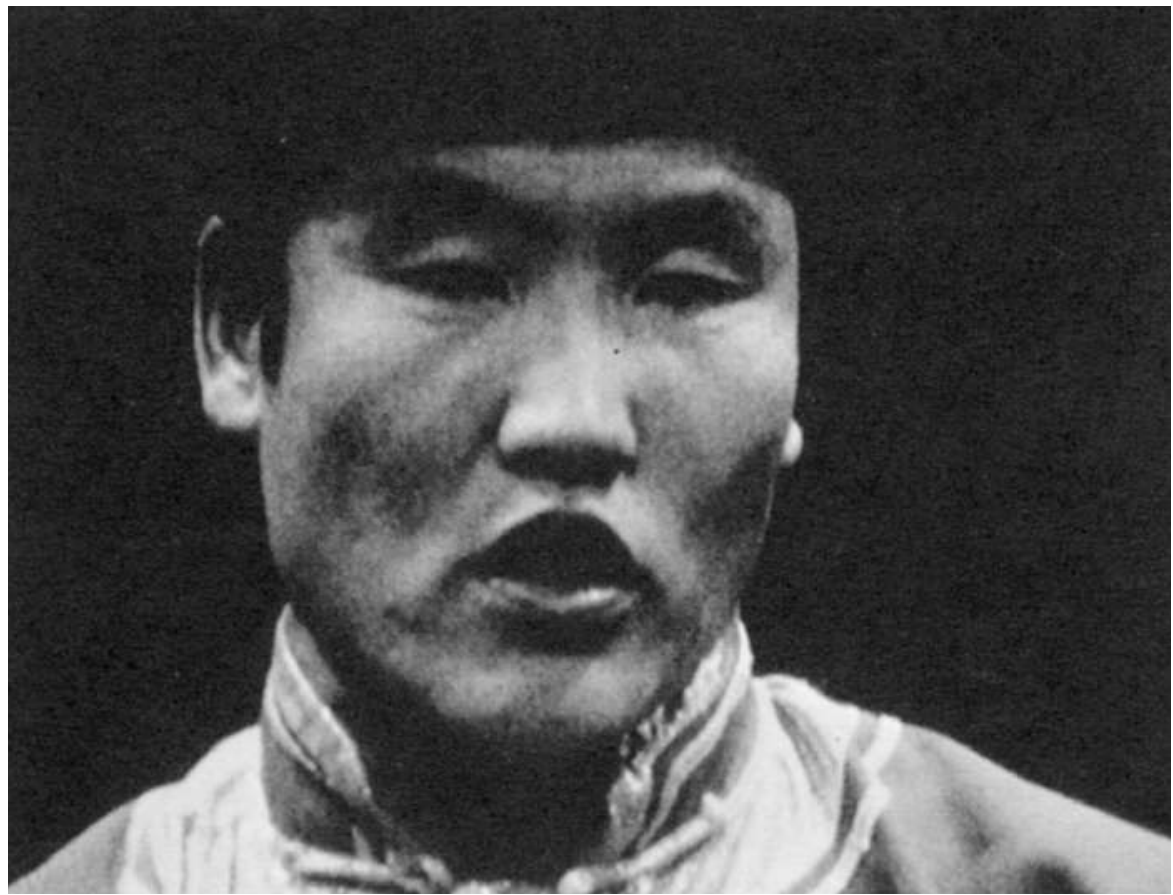

T. GANBOLD. Photogramme extrait du film «Le chant des harmoniques» de H. Zemp.

les plus aigus (180 et $220 \mathrm{~Hz}$ ) n'est pas plus élevée que la limite supérieure des harmoniques obtenus à partir du fondamental le plus grave, une octave plus bas (90 et $110 \mathrm{~Hz}$ ). On peut trouver la confirmation en examinant les fig. 3 et 4, reproduisant la voix de Minh-Tâm, la fille de Trân Quang Hai ${ }^{6}$. Avec la technique à une cavité et un fondamental de $240 \mathrm{~Hz}$, le nombre d'harmoniques est très restreint $\mathrm{H} 3$ à $\mathrm{H} 5(1200 \mathrm{~Hz})$. Avec la technique à deux cavités et un fondamental à $270 \mathrm{~Hz}$, les harmoniques $4(1080 \mathrm{~Hz})$ à $8(2160 \mathrm{~Hz})$ peuvent être utilisés pour créer une mélodie, ce qui donne une échelle plus riche (transposée do, mi, sol, sib-, do). Il s'en suit qu'une voix aiguë de femme ne permet pas de créer des mélodies selon la technique à une cavité. La femme xhosa d'Afrique du Sud enregistrée par le R.P. Dargie, qui utilise cependant cette technique (comme le montrent les fig. 7 et 8), a une voix grave, dans le registre des voix d'hommes (100 et $110 \mathrm{~Hz}=$ Sol1 et La1).

Si les conclusions que nous avons tirées de ces expérimentations (fig. 1 à 4) sont justes - et nous pensons qu'elles le sont - on devrait pouvoir en déduire que

6 Elle avait dix-sept ans quand cet enregistrement fut fait, mais son père lui avait enseigné le chant diphonique dès l'âge de six ans. 
les styles du chant diphonique dont les sonagrammes présentent une mélodie d'harmoniques ne dépassant pas pour l'essentiel $1 \mathrm{KHz}$ sont obtenus selon la technique à une cavité, alors que ceux dont la mélodie d'harmoniques se situe essentiellement entre 1 et $2 \mathrm{KHz}$ sont obtenus selon la technique à deux cavités. Les expériences faites par Trân Quang Hai, en essayant d'imiter les différentes variantes stylistiques, le confirment. Dans les lignes qui suivent, nous allons examiner les caractéristiques physiologiques des différentes variantes stylistiques du chant diphonique, en dégageant trois critères: le(s) résonateur(s); les contractions musculaires; les procédés d'ornementation.

\section{Le(s) résonateur(s)}

Selon Aksenov, le style kargiraa des Tuva se caractérise par un fondamental grave situé sur l'un des quatre degrés les plus bas de la grande octave, et pouvant descendre une tierce mineure pendant un court instant. Le changement mélodique d'un harmonique à l'autre est accompagné d'un changement de voyelles (Aksenov 1973: 13). Les deux chants reproduits ici (fig. 5, 9 et 10) ont des fondamentaux de $62 \mathrm{~Hz}$ et $67 \mathrm{~Hz}$ (momentanément $57 \mathrm{~Hz}$ ). La mélodie d'harmoniques atteint dans le premier cas $750 \mathrm{~Hz}$ (H12), dans le second cas 804 $\mathrm{Hz}(\mathrm{H} 12)^{7}$. Au dessus de la mélodie d'harmoniques on aperçoit une deuxième zone, à l'octave quand les voyelles postérieures sont prononcées (fig. 5 et 9), ou plus haut avec les voyelles antérieures (fig. 10) ${ }^{8}$. En imitant le tracé de la fig. 5, Trân Quang Hai utilise la technique à une cavité, la bouche semi-ouverte; à la différence des chants tuva, la mélodie d'harmoniques est à bande large, et H1 est très marqué (fig. 6). Tous les sept enregistrements identifiés sur les notices des disques comme faisant partie du style kargiraa chez les Tuva et karkira chez les Altaï ont été vérifiés au Sona-Graph ${ }^{9}$. La fréquence du fondamental se situe entre 62 et $95 \mathrm{~Hz}$; les mélodies d'harmoniques de toutes les pièces sans exception se situent en dessous de $1 \mathrm{KHz}$.

Ce n'est que depuis les travaux récents concernant la musique du peuple xhosa d'Afrique du Sud effectués par le R.P. Dargie (1989), et les enregistrements qu'il avait confiés en 1984 à Trân Quang Hai pour les archives sonores du

\footnotetext{
Selon Alekseev, Kirgiz et Levin (1990), la pièce que nous avons représentée dans les fig. 9 et 10 est une variante du kargiraa, appelée «Steppe kargiraa», et rappelle les chants tantriques des moines tibétains. Pour notre part, nous n'avons pas remarqué de différence avec les autres pièces de style kargiraa tuva, et la mélodie d'harmoniques très caractéristique n'a que peu d'éléments en commun avec les harmoniques qui apparaissent dans les chants tibétains des monastères Gyüto et Gyümë.

8 Les harmoniques de cette deuxième zone enrichissent sans doute le timbre, mais ils ne sont pas perçus par l'oreille comme formant une mélodie séparée de la mélodie d'harmoniques de la première zone.

9 Folkways/Smithsonian $n^{\circ}$ 1, 8, 9, 17, 18. Melodia, face A, plage 9. Le Chant du Monde, face A, plage 5 .
} 
Musée de l'Homme, que l'on connaît l'existence du chant diphonique pratiqué loin d'Asie centrale. La pièce chantée par une femme xhosa (fig. 7), caractérisée par l'alternance de deux fondamentaux de 100 et $110 \mathrm{~Hz}$ et d'une mélodie d'harmoniques ne dépassant pas $600 \mathrm{~Hz}$, est sans doute faite selon la technique à une cavité, comme le montre l'imitation de Trân Quang Hai aussi imparfaite qu'elle soit (fig. 8).

Qu'en est-il du style kargiraa khöömii ${ }^{10}$ du chanteur mongol T. Ganbold (fig. 11), dont nous avons placé le sonagramme à dessein en face d'un kargiraa tuva (fig. 9 et 10) ? Le fondamental est grave $(85 \mathrm{~Hz})$ et peu marqué comme dans les pièces tuva, mais la mélodie d'harmoniques se situe dans la zone de 1 à $2 \mathrm{KHz}$ et non pas au-dessous de $1 \mathrm{KHz}$. Pour obtenir un tracé semblable, Trân Quang Hai a dû employer la technique à deux cavités. Comment expliquer cette différence par rapport au style équivalent chez les Tuva? A l'arrivée de l'Ensemble de Danses et de Chants de la R.P. de Mongolie à Paris, l'Ambassade de Mongolie a organisé une réception à laquelle nous avons eu l'honneur et le plaisir d'assister. Comme d'autres artistes de la troupe, T. Ganbold y faisait une démonstration de son art, et nous avons pensé qu'il serait intéressant d'inclure dans notre film la pièce qu'il avait composée, «Liaisons de khöömii », pour présenter au public trois variantes stylistiques du chant diphonique ${ }^{11}$. La pièce ne figurait pas dans le programme des concerts à la Maison des Cultures du Monde, parce que T. Ganbold, l'ayant composée récemment, ne la maîtrisait pas encore complètement. En accord avec le directeur de la troupe, il a néanmoins accepté de la chanter sur scène hors concert pour le tournage du film, puis lors d'un concert, en bis (afin que nous puissions filmer son entrée sur scène ainsi que les applaudissements), et de faire une courte démonstration des différents styles lors de l'interview. En l'absence d'enregistrements d'autres chanteurs mongols, on ne peut dire si T. Ganbold s'est trompé de technique, ou si en Mongolie il est considéré comme juste de chanter le style khöömi kargiraa selon la technique à deux cavités. On peut aussi considérér que les frontières entre les différents styles ne sont pas rigides, que les chanteurs utilisent les possibilités techniques comme ils veulent (ou peuvent), qu'ils emploient les dénominations avec plus

10 La dénomination xaarkiraa xöömij est «traduite» dans Emmert et Minegushi (1980 48) par «narrative xöömij», sans doute pour rendre compte du fait que dans ce style la partie diphonique peut être précédée par une partie narrative chantée avec la voix naturelle. Dans le film Le chant des harmoniques, la dénomination kargiraa khöömii a été traduite par Alain Desjaques par «khöömii grue noire», khargiraa désignant en mongol cet oiseau dont la voix grave et enrouée aurait pu donner le nom à ce style. A moins que les Mongols aient emprunté le terme kargiraa aux Tuva pour qui, selon Alekseev, Kirgiz et Levin (1990), il proviendrait d'un mot onomatopéique signifiant «respirer péniblement», "parler d'une voix rauque ou enrouée». Nous ne pouvons trancher entre les deux interprétations. Il est aussi possible que les uns aient emprunté le terme aux autres, tout en lui trouvant postérieurement une signification dans leur propre langue.

11 Cela semble être un phénomène récent. Selon Alekseev, Kirgiz et Levin (1990), les chanteurs tuva étaient autrefois spécialisés en un ou deux styles apparentés, mais aujourd'hui les jeunes utilisent plusieurs styles et arrangent fréquemment des ségments mélodiques en des mélanges polystylistiques. 
ou moins de rigueur. Chez les Tuva aussi, nous le verrons dans le paragraphe suivant, une même dénomination de style peut désigner des chants exécutés selon les deux techniques différentes.

Selon Aksenov, le style borbannadir tuva se caractérise par un fondamental un peu plus élevé que celui du kargiraa, utilisant l'un des trois degrés au milieu de la grande octave. Les lèvres sont presque complètement fermées, le son en serait plus doux (soft) et résonnant. Le style borbannadir serait considéré par les Tuva comme techniquement similaire au style kargiraa, ce qui permettrait un changement subit de l'un à l'autre au sein d'une même pièce (1973: 14). L'examen des cinq pièces identifiées sur les notices de disques comme faisant partie du style borbannadir montre que dans deux pièces, le fondamental est à 75 et à $95 \mathrm{~Hz}$, et la mélodie d'harmoniques au-dessous de $1 \mathrm{KHz}^{12}$ (cf. fig. 13). Ces deux pièces sont donc effectivement très proches du style kargiraa, chanté selon la technique à une cavité. Les trois autres enregistrements de borbannadir ont un fondamental plus aigu $(120,170$ et $180 \mathrm{~Hz})$ et une mélodie d'harmoniques au-dessus de $1 \mathrm{KHz}$, donc obtenue par la technique à deux cavités ${ }^{13}$. Nous en reproduisons un exemple (fig. 15), et son imitation par Hai (fig. 16).

L'analyse sonagraphique montre (mais la simple écoute aussi) que dans un enregistrement dénommé borbannadir par les auteurs de la notice du disque, et dont nous avons déjà parlé brièvement (fig. 13), trois styles sont chantés en alternance. L'extrait à gauche présente toutes les caractéristiques du style kargiraa, qui est suivi manifestement, après une interruption d'une seconde, par le style borbannadir avec le même fondamental et la mélodie d'harmoniques au-dessous de $1 \mathrm{KHz}$. Le sonagramme de la fig. 14, montrant un autre extrait du même enregistrement, présente également à gauche le style kargiraa, suivi cette fois-ci sans interruption par un très court fragment ( 2 secondes) de borbannadir avec le même fondamental. Puis, après une courte interruption, le fondamental fait un saut d'octave de $95 \mathrm{~Hz}$ à $190 \mathrm{~Hz}$, et la mélodie d'harmoniques est située au-dessus de $1 \mathrm{KHz}$, dépassant même à certains endroits les $2 \mathrm{KHz}$ (cf. aussi les imitations de Hai, fig. 16 et 17). A l'écoute et sur le tracé du sonagramme, cette dernière partie ressemble beaucoup au style «khöömi de ventre» du chanteur mongol D. Sundui (fig. 20), et il n'est pas douteux qu'elle soit chantée selon la technique à deux cavités.

D'après Aksenov, dans certains lieux tuva, le nom de khomei remplace le nom de borbannadir. Parmi les six exemples de khomei tuva (dans le sens restreint) et le seul exemple de kiomioi altaï que nous connaissons, l'un a un fondamental grave de $90 \mathrm{~Hz}$ et des harmoniques ne dépassant pas $1 \mathrm{KHz}^{14}$, les quatre autres ont des fondamentaux entre $113 \mathrm{~Hz}$ et $185 \mathrm{~Hz}^{15}$ (fig. 18, et l'imita-

\footnotetext{
Folkways/Smithsonian, $\mathrm{n}^{\circ} 11$ et 14 .

Melodia, face 1, plage 5. Folkways/Smithsonian, no 12 et 13.

Folkways/Smithsonian, $n^{\circ} 7$.

15 Folkways/Smithsonian, no 5, 6, 8. Le Chant du Monde, face A, plage 4.
} 
tion fig. 19) et sont proches du borbannadir à fondamental aigu (cf. fig. 15 et 22) chanté selon la technique à deux cavités.

Le style ezengileer - dont un seul enregistrement est connu (fig. 21) - semble également proche du borbannadir à fondamental aigu (fig. 15 et 22).

Tous les enregistrements de borbannadir, khomei et ezengileer tuva que nous avons pu examiner ont en commun une pulsation rythmique que nous examinerons plus loin sous la rubrique des procédés d'ornementation. Il semble donc que pour le borbannadir, l'usage actuel permette deux variantes: un fondamental relativement grave ( 75 à $95 \mathrm{~Hz}$ ) et une mélodie d'harmoniques au-dessous de $1 \mathrm{KHz}$, donc chantée selon la technique à une cavité, et un fondamental plus aigu (120 à 190 Hz) avec une mélodie d'harmoniques au-dessus de $1 \mathrm{KHz}$, chantée selon la technique à deux cavités, le trait commun étant la pulsation rythmique.

Le style tuva qui s'oppose le plus clairement au kargiraa et au borbannadir (à fondamental grave) est le sigit. Selon Aksenov, il se caractérise par un fondamental plus tendu et plus élevé, la hauteur se situant au milieu de la petite octave. Le fondamental peut changer à l'intérieur d'une pièce et peut constituer la voix mélodique sans mélodie d'harmoniques au début des vers. A la différence des autres styles tuva, la voix supérieure ne constitue pas une mélodie bien caractérisée, mais reste longtemps sur une seule hauteur avec des ornements rythmiques (Aksenov 1973: 15-16). Cf. infra notre analyse des procédés d'ornementation.

Dans les onze enregistrements de sigit tuva et le seul exemple de sibiski altaï que nous connaissons, le fondamental se situe entre 160 et $210 \mathrm{~Hz}^{16}$. Nous avons choisi d'en reproduire trois sonagrammes (fig. 23, 24 et un bref extrait fig. 18). Pour l'imitation (fig. 26), Trân Quang Hai emploie la technique à deux cavités.

De nombreux chants mongols connus par des enregistrements ont une sonorité proche de celle du sigit. Le tracé des harmoniques est semblable, mais le style musical est différent en ce que les harmoniques font une véritable mélodie. C'est le cas des chants de D. Sundui (fig. 25), le spécialiste du chant diphonique mongol apparaissant probablement le plus souvent sur des disques ${ }^{17}$. Les notices de ces différents disques n'indiquent que le terme général (khöömii), mais on sait par ailleurs qu'il chante surtout le kevliin khöömii («khöömii de ventre»); ce dernier style et le tseedznii khöomii («khöömii de poitrine») étant pour lui «en général la même chose» (Emmert et Minegushi 1980: 48).

Dans l'interview du film Le chant des harmoniques, T. Ganbold appelle son style favori, dont un court exemple est reproduit en fig. 29, kholgoï khöömii («khöömii de gorge $)^{18}$; alors que pour l'enregistrement d'un disque effectué

16 Folkways/Smithsonian, $\mathrm{n}^{\circ}$ 2, 3, 4, 8, 16, 17. Melodia, face A, plages 1, 2, 6, 7, 8. Le Chant du Monde, face A, plage 3.

17 Tangent TGS 126, face B, plage 1 Tangent TGS 127, face B, plage 3. Victor, face A, plages 5, 6 et 7.

18 Selon Alain Desjacques qui a assumé la traduction, le terme kholgoï est synonyme de bagalzuuliin. 
trois jours auparavant, il a nommé ce même style tseedznii khöömii («khöömii de poitrine») ${ }^{19}$. S'est-il trompé lors de l'enregistrement du disque ou lors du tournage du film? Quoi qu'il en soit, le fait de se tromper confirme ce que nous avons déjà suggéré plus haut, à savoir que l'attribution d'une dénomination à un style ou à une technique ne semble pas être une préoccupation majeure pour certains chanteurs ${ }^{20}$. Cependant, T. Ganbold ayant été un élève de D. Sundui qui pratique le «khöömii de ventre» (ou de poitrine), nous sommes enclin à penser que «khöömii de poitrine» est le terme juste. L'examen des différences relatives aux contractions musculaires confirme cette hypothèse (cf. infra). Les autres enregistrements publiés de chant diphonique mongol, dont nous ne reproduisons pas ici des sonagrammes parce que le style n'est pas nommé sur les notices des disques, apparaissent appartenir à ce même style que D. Sundui appelle (rappellons-le) «khöömii de ventre», et qui semble être le plus répandu en Mongolie ${ }^{21}$.

Très proche du sonagramme du chant de D. Sundui est le tracé de l'enregistrement que John Levy a effectué en 1967 au Rajasthan (fig. 27). Aucune documentation concernant le lieu exact, le nom du chanteur et les circonstances de l'enregistrement n'accompagnant la bande magnétique déposée aux archives sonores du Musée de l'Homme, on en est réduit aux conjectures. Il est troublant qu'aucun enregistrement d'un autre chanteur du Rajasthan ne soit connu et qu'aucune publication ne mentionne le chant diphonique dans cette région. Lors de sa visite au Musée de l'Homme en 1979, Komal Kothari, directeur du Rajasthan Institute of Folklore, affirma à Trân Quang Hai qu'il avait entendu parler de ce phénomène vocal sans avoir pu l'écouter lui-même.

L'unique exemple de «khöömii de nez» dont nous disposons est un très court fragment de six secondes enregistré lors de l'interview avec T. Ganbold. La seule différence avec le «khöömii de poitrine» ( ?) (cf. fig. 29 et 31) tient au fait que le chanteur ferme complètement la bouche; la mélodie d'harmoniques est alors moins marquée, «noyée» en quelque sorte dans les harmoniques présents sur toute l'étendue du spectre (fig. 30 et 32).

Un dernier style reste à examiner le «khöömii de gorge» (nous corrigeons la dénomination, cf. supra) chanté par T. Ganbold (fig. 33, et son imitation par Trân Quang Hai, fig. 34). Il fait clairement partie des styles utilisant la technique à deux cavités. Nous en reparlerons sous la rubrique des contractions musculaires.

Selon Alain Desjacques qui a assumé la traduction, le terme kholgoï est synonyme de bagalzuuliin.

19 Cf. la notice du CD Maison des cultures du monde, $n^{\circ} 4$ et 6.

20 Un autre chanteur mongol a indiqué le nom de khooloin khöömii («khöömii de gorge») pour une pièce, et tseesni khendi (traduit par «technique ventrale») pour une autre, alors que la simple écoute montre, et l'analyse spectrographique le confirme, qu'il s'agit d'un même style (CD GREM, nº 32 et 33), en l'occurrence le style que D. Sundui appelle kevliin khöömii («khöömii de ventre»).

${ }^{21}$ Vogue, face B, plage 3. Hungaroton, face A, plage 5, et face B, plage 7. ORSTOM-SELAF, face B, plage 2. Maison des Cultures du Monde, $\mathrm{n}^{\circ}$ 4, 5, 6 . 
Le tableau 1 classe les différents styles selon les résonateurs.

\begin{tabular}{ll}
\hline 1 cavité & kargiraa (tuva) \\
& khargyraa khöömii (mongol) \\
& karkira (altaï) \\
& umngqokolo ngomqangi (xhosa) \\
& chants bouddhiques tibétains (monastère Gyüto) \\
& borbannadir et khomei grave (tuva) \\
& sigit (tuva) \\
& sibiski (altaï) \\
& chant du Rajasthan \\
& khöömii de poitrine (mongol) \\
& khöömii de ventre (mongol) \\
& khöömii de nez (mongol) \\
& khöömii de gorge (mongol) \\
& borbannadir et khomei aigu (tuva) \\
& kiomioi (altaï) \\
& ezengileer (tuva)
\end{tabular}

Tableau 1: Classement des styles en fonction des résonateurs.

\section{Contractions musculaires}

Lors des tournées européennes de chanteurs diphoniques mongols et tuva, Trân Quang Hai a souvent assisté aux concerts dans les coulisses ou rejoint les artistes dans les vestiaires. Il a pu constater que ceux-ci revenaient essoufflés et fatigués, le visage marqué par l'afflux du sang. Ce n'est certainement pas sans raisons que les pièces sont très courtes, et qu'un même chanteur n'interprète généralement pas plus de deux ou trois chants diphoniques lors d'un même programme.

En essayant de reproduire la sonorité des chants ainsi que le tracé des sonagrammes des différents styles du chant diphonique, Trân Quang Hai a remarqué qu'il devait employer différents degrés de contraction des muscles abdominaux et sterno-cléido-mastoïdiens (muscles du cou), et plus particulièrement au niveau du pharynx.

Dans le style kargiraa tuva et mongol, les muscles abdominaux et le pharynx sont relaxés (fig. 6 et 12). A première vue, cela n'a rien d'étonnant, puisque le kargiraa est le style dans lequel le fondamental est le plus grave (entre 57 et $95 \mathrm{~Hz}$ ), et pour produire un son grave il semble plus naturel de relâcher les muscles que de les contracter. Cependant, pour imiter le mieux possible le chant xhosa d'Afrique du Sud qui utilise également un fonda- 
mental grave (100 et 110 Hz), Trân Quang Hai a dû contracter très fortement les muscles abdominaux et le pharynx (fig. 8). Pour les chants mongols et tuva caractérisés par des fondamentaux plus aigus (entre 160 et $220 \mathrm{~Hz}$ ), l'expérience montre des degrés variables de la tension musculaire. Ainsi, la plus forte contraction apparaît dans le style sigit tuva (fig. 26), avec un conduit d'air étroit. En sont très proches le chant du Rajasthan (fig. 28), le style «khöömii de poitrine» ( ?) mongol de T. Ganbold (fig. 29) et le «khöömii de ventre» de D. Sundui (fig. 25). Ce dernier, lors d'un festival de musique asiatique en Finlande $^{22}$, a pris la main de Trân Quang Hai pour la poser successivement sur son ventre et sur sa gorge, afin de lui faire ressentir les différences de contraction. Pour le khargiraa khöömii, les muscles abdominaux étaient relâchés; pour le «khöömii de ventre» (la spécialité de D. Sundui), le ventre était dur comme de la pierre. Est-ce la raison de cette dénomination? Si, comme dit D. Sundui, «khöömii de ventre» et «khöömii de poitrine»sont en général la même chose (op. cit.), comment expliquer les deux expressions ? Quand Trân Quang Hai imite ce style, il ressent une vibration en haut de la poitrine, au niveau du sternum. La dénomination «khöömii de ventre» se référerait alors à la très grande tension des muscles abdominaux; la dénomination «khöömii de poitrine» indiquerait plutôt la vibration que le chanteur ressent au niveau du sternum. L'autre style utilisé par T. Ganbold, dont il faudrait alors corriger l'appellation en «khöömii de gorge» (et non pas «de poitrine»comme il dit dans le film), est caractérisé par une mélodie d'harmoniques beaucoup moins marquée, «noyée» en quelque sorte dans les harmoniques couvrant toute l'étendue du spectre (fig. 33). En essayant de l'imiter (fig. 34), Trân Quang Hai contracte moins les muscles abdominaux et le pharynx, et quand on pose les doigts sur la gorge au-dessus du cartilage thyroïde (pomme d'Adam), on perçoit effectivement une vibration plus forte à ce niveau-là.

Dans le borbannadir à fondamental aigu (fig. 20) et le khomei (fig. 19), les contractions musculaires semblent plus faibles que dans le sigit, mais plus fortes que dans le kargiraa.

La contraction musculaire est-elle un facteur déterminant du style ou de la manière de chanter d'un individu ${ }^{23}$ ? Trân Quang Hai réussit à imiter les caractéristiques stylistiques du sigit tuva ou du «khöömii de ventre» mongol sans contraction abdominale et pharyngienne excessive, mais la puissance de la mélodie d'harmoniques est nettement moindre et la sonorité plus matte (fig. 37). Pour produire une plus grande puissance et un son ressemblant aux enregistrements des chanteurs tuva et mongols, il contracte à l'extrême les muscles abdominaux et sterno-cléido-mastoïdiens du cou. En bloquant le pharynx pour obtenir un conduit d'air très resserré, il obtient une mélodie

\footnotetext{
22 Trân Quang Hai était directeur artistique pour la section de musique asiatique au festival de musique de chambre à Kuhmo en 1984, et à ce titre avait invité le chanteur D. Sundui à se produire en Finlande.

23 Nous devons cette question à Gilles Léothaud qui l'a posée au séminaire de notre équipe de recherche lorsque nous avons exposé nos travaux.
} 
d'harmoniques plus «détachée» des autres harmoniques du spectre (fig. 35). En contractant un peu moins le pharynx et en laissant un conduit d'air plus large, il a l'impression d'avoir davantage de résonance dans les cavités buccales; le résultat est une mélodie d'harmoniques plus large et un tracé plus foncé de l'ensemble des harmoniques (fig. 36). A titre comparatif, nous avons reproduit un sonagramme montrant l'émission diphonique à bouche fermée (fig. 38).

Le premier à avoir suggéré une relation entre la tension de certaines parties corporelles et la brillance des sons harmoniques dans le chant diphonique est $\mathrm{S}$. Gunji. S'appuyant sur un texte de l'acousticien allemand F. Winckel, il rappelle que les parois intérieures des cavités corporelles sont molles et peuvent être modifiées par tension; si la tension est élevée, les hautes fréquences ne seront pas absorbées et le son sera très brillant, et le contraire survient si le degré de tension est bas (Gunji 1980: 136). Il faudrait poursuivre les recherches dans ce domaine - y compris avec des chanteurs d'opéra qui obtiennent de la puissance et des sons brillants apparemment sans tension musculaire excessive - avant de pouvoir tirer des conclusions définitives.

Le tableau 2 classe les styles en fonction de leur contraction musculaire.

Relaxation pharyngienne et abdominale kargiraa (fig. 5, 9, 10, imitation 6)

kargiraa khöömii (Ganbold, fig. 11, imitation fig. 12) karkira (altaï) chant bouddhique tibétain (monastère Gyütö) borbannadir grave (fig. 13, imitation fig. 16). borbannadir aigu (fig. 15 et 22, imitation fig. 20) khomei (fig. 18, imitation fig. 19) khöömii de gorge (?) Ganbold, fig. 33, imitation fig. 34)

sigit (fig. 23 et 24, imitation fig. 26)

sibiski (altaï)

khöömii de poitrine (?) (Ganbold fig. 29)

khöömii de ventre (fig. 25)

khöömii de nez (fig. 30)

umngqokolo ngomqangi (fig. 7, imitation fig. 8)

Tableau 2: Classement des styles en fonction de la contraction musculaire. 


\section{Procédés d'ornementation}

Les enregistrements sonores dont nous disposons présentent plusieurs procédés d'ornementation qui enrichissent la texture rythmique et harmonique du chant diphonique. La réduction du format des sonagrammes - nécessaire pour cette publication - rend la lecture des ornements difficile. Nous avons choisi de reproduire à une plus grande échelle huit extraits de chants déjà analysés: cette fois-ci l'analyse de la fréquence est limitée à $2 \mathrm{KHz}$ (et non pas à $4 \mathrm{KHz}$ ), et l'axe temporel est deux fois plus grand (fig. 39 à 42).

1) Dans le style sigit tuva et sibiski altaï, il ne s'agit pas d'une ornementation ajoutée à la mélodie, mais de l'élément principal du style musical. Comme le dit Aksenov, à la différence des autres styles tuva, la voix supérieure du sigit ne constitue pas une mélodie bien caractérisée mais plutôt un rythme ponctué principalement sur deux hauteurs, le $9^{\mathrm{e}}$ et le $10^{\mathrm{e}}$ harmoniques des deux fondamentaux $^{24}$ (Aksenov 1973: 15-16). Ces ponctuations se succèdent à un rythme régulier une fois par seconde ou un peu plus rapproché (fig. 23, 24 et 39).

Logiquement, pour obtenir une ponctuation sur l'harmonique immédiatement supérieur à la ligne mélodique principale, il faut diminuer le volume de la cavité buccale antérieure en avançant la pointe de la langue. Avancer très rapidement et reculer une fois par seconde la pointe de la langue, qui est dirigée verticalement contre le palais, est assez inconfortable. Hai a trouvé une autre possibilité en aplatissant légèrement la pointe de la langue contre le palais, la cavité buccale antérieure est également raccourcie, et en revenant rapidement dans la position initiale, le mouvement de la langue est plus confortable (fig. 26). On ne sait pas comment les chanteurs du sigit procèdent, si c'est la première ou la deuxième solution qu'ils adoptent, mais selon la loi du moindre effort, on peut supposer qu'ils utilisent le second procédé. Sur le sonagramme, cette ponctuation est le très marquée sur l'harmonique immédiatement supérieur de la ligne mélodique, et plus faiblement sur le deuxième et le troisième harmoniques au dessus, mais pas sur les harmoniques inférieurs, notamment ceux du bourdon.

2) Un autre procédé de pulsation rythmique est effectué sans aucun doute par des coups de langue dirigés verticalement contre le palais. Nous en connaissons deux exemples, l'un provenant de Mongolie ${ }^{25}$, l'autre du Rajasthan (fig. 27 et 40). Cette fois-ci, l'accentuation est visible sur toute l'étendue du spectre sonore, et en particulier sur $\mathrm{H} 2$ et 3 par des traits verticaux. La langue n'est pas avancée horizontalement ou aplatie contre le palais comme dans le sigit, mais elle fait un mouvement de va-et-vient vertical (imitation fig. 28).

Le tracé de la partie droite de la fig. 15, représentant un borbannadir, montre sur $\mathrm{H} 13$ une ponctuation rythmique proche de celle du sigit, et une

\footnotetext{
24 «an ornamented trilling and punctuating rhythm principally on two pitches (the ninth and tenth partials of the two fundamentals)»

25 GREM, no 2 .
} 
accentuation sur les harmoniques inférieurs, notamment sur $\mathrm{H} 3$, ce qui laisse supposer qu'elle est faite par des coups de langue (fig. 20) comme dans le cas du chant du Rajasthan.

3) Selon Aksenov, dans le style ezengileer, caractérisé par des accents irréguliers (agogic), on entend dans la mélodie des harmoniques et dans le fondamental les pulsations dynamiques ininterrompues du rythme du galop, la dénomination de ce style venant du terme «étrier» (1973: 16). Aksenov mentionne ailleurs que le chant du borbannadir peut être ininterrompu ou brisé; dans ce dernier cas, l'intonation du $\underline{\mathbf{v}}$ est interrompue par la fermeture des lèvres suivie par leur ouverture, ou bien sur $\underline{x}$, la consonne occlusive $\underline{b}$ ou la consonne nasale $\underline{\mathrm{m}}^{26}$. Alekseev, Kirgiz et Levin (1990) écrivent que ce rythme pulsé et asymétrique du ezengileer est produit par de rapides vibrations des lèvres, et que le terme de borbannadir - «utilisé métaphoriquement pour signifier "roulant" »- est caractérisé par le même rythme pulsé ${ }^{27}$.

Cette pulsation est visible sur les sonagrammes du khomei et du ezengileer par un tracé sur une ou deux hauteurs d'harmoniques, alternativement audessus et au-dessous de la ligne horizontale. Dans l'exemple khomei (fig. 18 et 45), il y a cinq battements par seconde; dans l'exemple ezengileer (fig. 21 et 46), les battements sont plus rapprochés: neuf par seconde. Pour obtenir un tracé semblable, Trân Quang Hai fait de rapides mouvements de la lèvre inférieure vers la lèvre supérieure (fig. 19). Sur les sonagrammes du borbannadir à fondamental grave (fig. 13 et 43), la pulsation à huit battements/seconde est reproduite en forme de «zigzag»; pour l'imiter, Trân Quang Hai fait des mouvements rapides de la langue d'avant en arrière (fig. 16).

4) $\mathrm{Si}$ les ornements et procédés rythmiques décrits dans les paragraphes 1 à 3 sont exclusivement réalisés par l'appareil phonatoire, un autre procédé utilise une intervention extérieure: le frappement d'un doigt (des doigts) entre (contre) les lèvres ${ }^{28}$ (Alekseev, Kirgiz et Levin 1990), marqué sur les sonagrammes (fig. 22 et 44) par une sorte de «hachure» sur H2. Comme ces auteurs ne donnent pas plus de précisions, Trân Quang Hai a essayé toutes les variantes possibles de frappement, avec un ou plusieurs doigts, en position horizontale ou verticale, contre ou entre les lèvres. Mais aucun de ces essais ne permettait

${ }^{26}$ In the broken singing of this style the intoning of $\underline{v}$ is interrupted by the full closing of the lips followed by opening either on $\underline{x}$ the plosive voiced consonant $\underline{b}$ or on the nasal consonant $\underline{m}$. (Aksenov 1973: 14)

27 Les trois auteurs écrivent que la mélodie d'harmoniques est produite par de rapides vibrations de lèvres (In ezengileer, soft, shimmering harmonic melodies produced by rapide vibrations of the lips, are sung over a low fundamental drone). Le lecteur corrigera de lui-même cette mauvaise formulation, car bien entendu ce n'est pas la mélodie d'harmoniques qui est produite par la vibration des lèvres, mais le rythme pulsé. De même, la formulation disant que le style «borbannadir est utilisé métaphoriquement pour signifier "roulant" » (borbannadir, used metaphorically to signify "rolling”) n'est pas très heureuse.

28 C'est ainsi qu'on peut traduire l'expression «with finger strokes across the lips». On peut supposer qu'un doigt (l'index?) est mis horizontalement entre les lèvres, et qu'un rapide vaet-vient vertical imprime la pulsation à l'émission diphonique. 
de différencier le rythme irrégulier du frappement de(s) doigt(s) sur H2, de la pulsation régulières des harmoniques supérieurs.

5) Il n'est peut-être pas orthodoxe d'inclure le vibrato dans les procédés d'ornementation (encore que certains musicologues le font), mais il nous semble intéressant de l'étudier ici précisément pour dégager les différences mais aussi les similitudes avec les autres techniques. En fin de compte, c'est un procédé comme les autres pour enrichir la sonorité du chant diphonique et structurer le temps.

Le vibrato en modulation de fréquence est caractérisé sur le sonagramme par une fluctuation plus ou moins forte de la mélodie d'harmoniques principale ainsi que des autres harmoniques figurant sur le tracé, dessinant quatre à cinq «ondulations» par seconde. Le vibrato le plus fort que nous avons entendu et vu sur un sonagramme est fait par le chanteur mongol D. Sundui, dont le chant diphonique se caractérise par ailleurs par l'emploi d'une mélodie d'harmoniques à large bande: à certains endroits, le «sommet de la vague» d'un harmonique touche presque le «bas de la vague» de l'harmonique supérieur (fig. 25 et 41). Sur H8 par exemple, le vibrato oscille entre 1620 et $1790 \mathrm{~Hz}$, l'ambitus du vibrato étant de 170 cents, c'est-à-dire entre le trois-quart de ton et le ton entier. Cet artiste ayant étudié le chant classique occidental au conservatoire de $\operatorname{Moscou}^{29}$ - il chante d'ailleurs aussi selon la technique du chant diphonique des airs de compositeurs comme Tchaikovsky et Bizet (Batzengel 1980: 52) - on serait tenté de supposer que son vibrato très marqué ait été acquis lors de ses études au conservatoire ${ }^{30}$. Le chanteur du Rajasthan (fig. 27), dont on ne sait rien, a aussi un vibrato très fort, également combiné avec une mélodie d'harmoniques à large bande.

Le vibrato peut être combiné avec d'autres procédés d'ornementation, comme les coups de langue contre le palais dans le chant du Rajasthan (fig. 27), ou l'aplatissement rythmique de la langue contre le palais dans le sigit (fig. 26). Dans ce dernier exemple, on peut compter quatre oscillations de vibrato pour un accent rythmique par seconde. Le sonagramme de la fig. 18 est particulièrement intéressant puisqu'il permet de comparer le tracé du vibrato (à droite pour le sigit) avec la pulsation rythmique (à gauche pour le khomei), les deux procédés étant utilisés successivement par le même chanteur dans la même pièce.

Le tableau 3 résume ces différents procédés d'ornementation.

A quel point l'analyse spectrographique montre-t-elle des différences individuelles de l'émission vocale entre des chanteurs utilisant le même style? Cette question mériterait une analyse approfondie, mais avec beaucoup plus d'enregistrements d'un même style chanté plusieurs fois par le même chanteur, et par des chanteurs différents. L'examen des sonagrammes que nous avons

Communication verbale de D. Sundui à Trân Quang Hai lors du festival en Finlande, 1984.

30 Pour ce qui est du chant d'opéra et de concert, l'ondulation du vibrato est décrit comme presque sinusoïdale, avec un ambitus de l'ordre de +50 Hz (Sundberg 1980: 85), c'est-à-dire du quart de ton. 
Coups de langue

contre le palais

Aplatissement rythmique de la pointe de la langue contre le palais

Mouvements rapides

de fermeture et

d'ouverture des lèvres

Mouvements de va et vient horizontal de la langue

Frappements de(s) doigts entre (contre) les lèvres

Vibrato
Chant du Rajasthan (fig. 27, 40, imitation

fig. 28) borbannadir (fig. 15, imitation fig. 20)

sigit tuva (fig. 18, 23, 24, 39, imitation fig. 26) sibiski altaï

khomei (fig. 18 et 45, imitation fig. 19)

borbannadir à fondamental grave

(fig. 13 et 43, imitation fig. 16)

borbannadir (fig. 22 et 44)

plus ou moins présent dans la plupart

des chants les plus marqués:

fig. 5 et 42, 9 et 10, 25 et 41, 27

Tableau 3: Procédés d'ornementation.

effectués permet néanmoins de donner une première réponse: il apparaît qu'il y a en effet des différences individuelles (que l'on peut supposer être du même ordre que les différences de style et de voix des chanteurs d'opéra, par exemple). Nous avons vu que pour les quatre styles chantés par T. Ganbold, le spectre sonore est caractérisé par une zone importante d'harmoniques audessus de $4 \mathrm{KHz}$, ce qui n'est pas le cas chez les autres chanteurs dont nous publions des sonagrammes. Deux sonagrammes du style sigit chanté par deux chanteurs différents montrent chez l'un une sorte de «bourdon harmonique» au-dessus de la mélodie d'harmoniques (fig. 23), absent chez l'autre (fig. 24). De même, le «bourdon harmonique» est très marqué chez un chanteur de kargiraa (fig. 5), et absent chez un autre (fig. 9 et 10). Bien entendu, il faut être prudent et ne pas tirer des conclusions définitives avec si peu d'exemples, car surtout dans les zones aigües, certaines différences peuvent résulter également des conditions d'enregistrement (équipement, environnement).

Mais des différences se remarquent aussi dans des zones plus graves: sur beaucoup de sonagrammes, l'harmonique 1 est très faible (fig. 5, 7, 9, 10, 11, 13, 18, 22), alors que sur d'autres, il est au contraire très marqué (fig. 15, 21, 25, 27 , 29, 30, 33). L'intensité de H1 est-elle fonction du style? Nous ne le pensons pas: en tout cas, le style sigit peut manifestement comporter un H1 faible (fig. 18) 
ou fort (fig. 23 et 24). L'intensité de l'harmonique 1 semble être plutôt une caractéristique individuelle des chanteurs. Chez T. Ganbold, H1 est très marqué quel que soit le style (rappelons qu'il est le seul chanteur pour qui nous disposons d'enregistrements de quatre styles: fig. 11, 29, 30, 33). De même, tous les sonagrammes de chants de Trân Quang Hai ont l'harmonique 1 très marqué. Cependant, nous avons au moins un sonagramme montrant d'un même chanteur un H1 faible pour un style et un H1 fort pour un autre (fig. 14). Là encore, les recherches devraient être poursuivies.

Faut-il parler de «chant triphonique» quand des chanteurs font ressortir un deuxième bourdon soit dans l'aigu avec l'harmonique 18 (fig. 23) ou l'harmonique 44 (fig. 5), soit dans le grave avec l'harmonique 3 (fig. 15) ? Dans le premier cas, le sifflement aigu de ce «bourdon harmonique» peut difficilement être qualifié de troisième voix. Dans le deuxième cas, l'auteur de la notice en tout cas pense que dans le borbannadir en question, cette quinte qu'on entend très clairement au-dessus de l'octave du fondamental «fait naître comme résultat un chant à trois voix » ${ }^{31}$. Cependant, dans les deux cas, il n'y a pas de troisième voix indépendante du fondamental et de la mélodie d'harmoniques. Il s'agit manifestement de variantes individuelles que, pour ce qui est du bourdon à la quinte (H3), Trân Quang Hai imite bien (fig. 20). Ces chants sont de même nature que tous les autres analysés dans cet article, et nous ne pensons pas qu'il y ait lieu de changer de terminologie.

Nous avons gardé pour la fin deux sonagrammes représentant des exercices de style (et non pas des styles traditionnels!), où Trân Quang Hai fait preuve de toute sa virtuosité.

Dans la fig. 47, il inverse le dessin habituel d'un bourdon grave et d'une mélodie d'harmoniques dans l'aigu, pour en faire à partir de fondamentaux changeants une hauteur fixe dans l'aigu. Ainsi, le «bourdon harmonique» de $1380 \mathrm{~Hz}$ est réalisé successivement par H12, 9, 8, 6, 8, 9, 12, alors que les fondamentaux changent en montant $\mathrm{La} 1, \mathrm{Re} 2, \mathrm{Mi} 2, \mathrm{La} 2$ et en redescendant au La1.

Dans la fig. 48, l'exercice est encore plus difficile, puisqu'il présente simultanément une échelle ascendante pour les fondamentaux et une échelle descendante pour les harmoniques, et vice versa.

Reproduites ici pour l'intérêt scientifique (et le plaisir) qu'apporte l'exploration des limites physiologiques de la voix humaine, ces possibilités techniques ne sont pas exploitées dans les traditions d'Asie centrale et d'Afrique du Sud. Ne doutons pas qu'elles le seront bientôt par des compositeurs de musique contemporaine en Occident dont certains, depuis Stimmung (1968) de Stockhausen, cherchent à enrichir les techniques vocales par les moyens du chant diphonique.

31 Traduction de la notice de B. Tchourov par Philippe Mennecier (communication personnelle). Le même chanteur a exécuté deux pièces de styles sigit et deux pièces de style khomei qui ne comportent pas ce «bourdon à la quinte» (Melodia, face A, plages 1 à 4). 


\section{Bibliographie}

AKSENOV A. N.

1973 «Tuvin Folk Music». Asian Music 4(2): 7-18. (Extraits traduits de Tuvinskaia narodnaia muzyka, Moskou 1964)

ALEKSEEV Eduard, Zoya KIRGIZ et Ted LEVIN

1990 Notice du disque Tuva-Voices from the Center of Asia.

BATZENGEL

1980 «Urtiin duu, xöömij, and morin xuur». In R. Emmert et Y. Minegushi (ed.), Musical Voices of Asia 52-53. Tokyo Heibonsha Publishers.

BOREL-MAISONNY Suzanne et Michèle CASTELLENGO

1976 «Étude radiographique des mouvements oro-pharyingés pendant la parole et le jeu instrumental», Bulletin du Groupe d'Acoustique Musicale 86 (Université de Paris VI).

DARGIE David

1988 Xhosa Music. Its techniques and instruments, with a collection of songs. Cape Town et Johannesburg David Philip.

DESJACQUES Alain

1988 «Une considération phonétique sur quelques techniques vocales diphoniques mongoles». Bulletin du Centre d'Études de Musique Orientale 31: 46-45 (Paris).

EMMERT Richard et Yuki MINEGUSHI (ed.)

1980 Musical Voices of Asia. Tokyo Heibonsha Publishers.

GUNJI Sumi

1980 «An Acoustical Consideration of Xöömij». In R. Emmert et Y. Minegushi (ed.), Musical Voices of Asia 135-141. Tokyo Heibonsha Publishers.

HAMMAYON Roberte

1973 Notice du disque Chants mongols et bouriates. Collection Musée de l'Homme. Vogue LDM 30138.

HARVILAHTI Lauri

1983 «A two voiced-song with no words». Suomalais-ugrilaisen seuran aikakauskirja 78: 43-56 (Helsinki).

HARVILAHTI Lauri et KASKINEN H.

1983 «On the Application Possibilities of Overtone Singing». Suomen Antropologi i Finland 4: 249-255 (Helsinki).

LEIPP Émile

1971 «Le problème acoustique du chant diphonique». Bulletin du Groupe d'Acoustique Musicale 58: 1-10. Université de Paris VI.

LEOTHAUD Gilles

1989 «Considérations acoustiques et musicales sur le chant diphonique», Institut de la voix, Dossier $n^{\circ}$ 1: 17-44. Limoges.

PAILLER Jean-Pierre

1989 «Examens vidéo de Monsieur Trân Quang Hai». Institut de la voix, Dossier nº 1: 11-14. Limoges.

PETROV Valérii et Boris TIKHOMIROV

1985 Notice du disque Voyage en URRS vol. 10 Sibérie, Extrême Orient, Extrême Nord.

SAUVAGE Jean-Pierre

1989 «Observation clinique de Monsieur Trân Quang Hai». Institut de la voix, Dossier $n^{\circ} 1$ : 3-10. Limoges.

SUNDBERG Johan

1980 «Acoustics, IV The voice». In Stanley Sadie (ed.), The New Grove Dictionary of Music and Musicians 1: 82-87. Londres Macmillan. 
TRÂN Quang Hai

1989 «Réalisation du chant diphonique». Institut de la voix, Dossier n $n^{\circ} 1$ : 15-16. Limoges.

TRÂN Quang Hai et Denis GUILLOU

1980 «Original Research and Acoustical Analysis in connection with the Xöömi Style of Biphonic Singing». In R. Emmert et Y. Minegushi (ed.), Musical Voices of Asia : 162-173. Tokyo Heibonsha Publishers.

WALCOTT Ronald

1974 «The Chöömij of Mongolia A Spectral Analysis of Overtone Singing». Selected Reports in Ethnomusicology 2(1): 55-60.

ZEMP Hugo

1989 «Filming Voice Technique: The Making of "The Song of Harmonics"». The World of Music 31(3): 56-85.

\section{Discographie}

(Pour des raisons de commodité, les références dans le texte et dans les légendes concernent les éditeurs plutôt que les titres des disques dont plusieurs se ressemblent.)

GREM, G 7511. Mongolie. Musique et chants de tradition populaire. 1 disque compact. Enregistrements et notice de X. Bellenger. 1986.

Hungaroton, LPX 18013-14. Mongol Nepzene (Musique populaire mongole). 2 disques $30 \mathrm{~cm}$. Enregistrements et notice de Lajos Vargyas. Unesco Co-operation, ca. 1972. (Réédition Mongolian Folk Music, 2 disques compacts, HCD 18013-14, 1990).

Le Chant du Monde, LDX 74010. Voyage en URRS vol. 10 : Sibérie, Extrême Orient, Extrême Nord. 1 disque $30 \mathrm{~cm}$. Notice de V. Petrov et B. Tikhomirov. 1985.

Maison des Cultures du Monde, W 260009. Mongolie. Musique vocale et instrumentale. 1 disque compact, série «Inédit». Notice de P. Bois. 1989.

Melodia, GOCT 5289-68. Pesni i instrumental'nye melodii tuvy (Chants et mélodies instrumentales des Touva). 1 disque $30 \mathrm{~cm}$. Notice de B. Tchourov.

ORSTOM-SELAF, Ceto 811. Mongolie. Musique et chants de l'Altaï. 1 disque $30 \mathrm{~cm}$. Enregistrements et notice de Alain Desjacques. 1986.

Smithsonian/Folkways SF 40017. Tuva-Voices from the Center of Asia. 1 disque compact. Enregistrements et notice de E. Alekseev, Z. Kirgiz et T. Levin. 1990.

Tangent TGS 126 et 127. Vocal Music from Mongolia; Instrumental Music from Mongolia. 2 disques $30 \mathrm{~cm}$. Enregistrements et notices de Jean Jenkins. 1974.

Victor Records, SJL 209-11. Musical Voices of Asia (Asian Traditional Performing Arts 1978). 3 disques $30 \mathrm{~cm}$. Enregistrements de S. Fujimoto et T. Terao, notice de F. Koizumi, Y. Tokumaru et O. Yamaguchi. 1979.

Vogue LDM 30138. Chants mongols et bouriates. 1 disque $30 \mathrm{~cm}$. Enregistrements et notice de Roberte Hamayon. Collection Musée de l'Homme. 1973.

\section{Filmographie}

Le chant des harmoniques (version anglaise The Song of Harmonics). 16 mm, 38 min. Auteurs: Trân Quang Hai et Hugo Zemp. Réalisateur: Hugo Zemp. Coproduction CNRS Audiovisuel et Société Française d'Ethnomusicologie, 1989. Distribition CNRS Audiovisuel, 1, place Aristide Briand, F-92195 Meudon. 


\section{LECTURE DES SONAGRAMMES}

Dans le film Le chant des harmoniques, les sonagrammes en temps réels sont reproduits selon une échelle de couleurs qui nous semblait la plus lisible. Pour l'impression en noir et blanc de cette revue, nous avons choisi de photographier l'inscription sur papier continu que fournit le Sona-Graph, où les degrés de noirceur correspondent aux degrés d'intensité des harmoniques.

Les mélodies d'harmoniques ne dépassant jamais la limite supérieure de $3000 \mathrm{~Hz}$, l'analyse spectrographique dans cet article a été limitée à $4 \mathrm{KHz}$. Cependant, pour être sûr que d'éventuelles zones de formants dans des fréquences supérieures n'échappent à l'analyse, nous avons examiné tous les exemples choisis avec une grille jusqu'à $8 \mathrm{KHz}$. Seul le chanteur mongol T. Ganbold dépasse $4 \mathrm{KHz}$, non pas avec la mélodie d'harmoniques, mais avec une troisième zone de formants (affectant sans doute le timbre, mais non pas le contour de la mélodie d'harmoniques). Nous reproduisons les sonagrammes de deux enregistrements analysés d'abord à $4 \mathrm{KHz}$ (fig. 29 et 30), puis à $8 \mathrm{KHz}$ (fig. 31 et 32).

Les modèles précédents du Sona-Graph ne permettaient d'imprimer qu'une inscription correspondant à 2,4 secondes, respectivement à 4,8 secondes si la bande magnétique était lue à moitié vitesse. Le DSP Sona-Graph 5500 peut analyser et inscrire sur du papier continu jusqu'à 3 minutes. Pour reproduire les sonagrammes sur une page de revue, il nous a fallu choisir la durée maximale de l'enregistrement, l'étalement du signal en fonction du temps, tout en faisant attention à ce que les différents harmoniques soient lisibles. En imprimant, pour des raisons d'économie, deux sonagrammes sur une même page, complétés par des légendes substantielles, nous avons pensé qu'une durée maximale de 14 secondes constituait le meilleur compromis. Bien sûr, une reproduction plus grande donnerait une lisibilité accrue, mais étant donné qu'il ne fallait pas augmenter le nombre de pages de l'article ni les frais de photogravure, la réduction choisie nous a semblé acceptable. Pour faciliter la comparaison, la majorité des sonagrammes (fig. 3 à 38, 47 et 48) ont été reproduits à la même échelle. Si l'inscription couvre toute la largeur du «satzspiegel», il s'agit donc d'un signal de 14 secondes; si deux sonagrammes de longueur sensiblement égale se partagent la même surface, le signal est évidemment de moitié plus court, c'est-à-dire de 7 secondes environ. Par contre, l'inscription des deux premiers sonagrammes a dû être comprimée afin de pouvoir reproduire la totalité de l'échelle des fondamentaux (20 secondes pour la fig. 1, 28 secondes pour la fig. 2).

Pour des raisons de lisibilité, nous avons reproduit, à une échelle plus grande, huit extraits de chants déjà analysés (fig. 39 à 46) : cette fois-ci l'analyse de la fréquence est limitée à $2 \mathrm{KHz}$ (et non pas à $4 \mathrm{KHz}$ ), et l'axe temporel est deux fois plus grand, ce qui représente pour chaque sonagramme une durée de 3 secondes environ. 


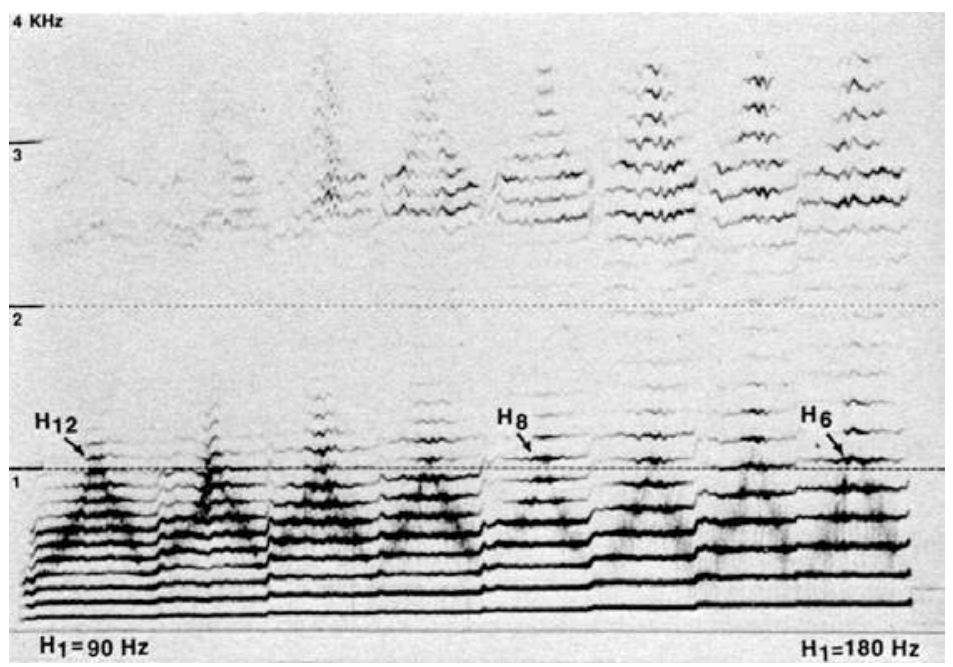

Fig. 1: Échelles d'harmoniques avec différentes hauteurs du fondamental (90 à $180 \mathrm{~Hz}$ selon une échelle diatonique), par Trân Quang Hai. Technique à une cavité.

Voix fortement nasalisée. Prononciation marquée de voyelles. H1, 2 et 3 du bourdon très marqués. Avec le fondamental le plus grave $(90 \mathrm{~Hz})$, les harmoniques $4(360 \mathrm{HZ})$ à $12(1080 \mathrm{~Hz})$ peuvent être utilisés pour créer une mélodie. Avec le fondamental le plus aigu $(180 \mathrm{~Hz})$, seuls les harmoniques 3 à $6(1080 \mathrm{~Hz})$ ressortent. Deuxième zone d'harmoniques entre 2500 et $4000 \mathrm{~Hz}$, séparée des harmoniques utilisables par une «zone blanche» sur le sonagramme.

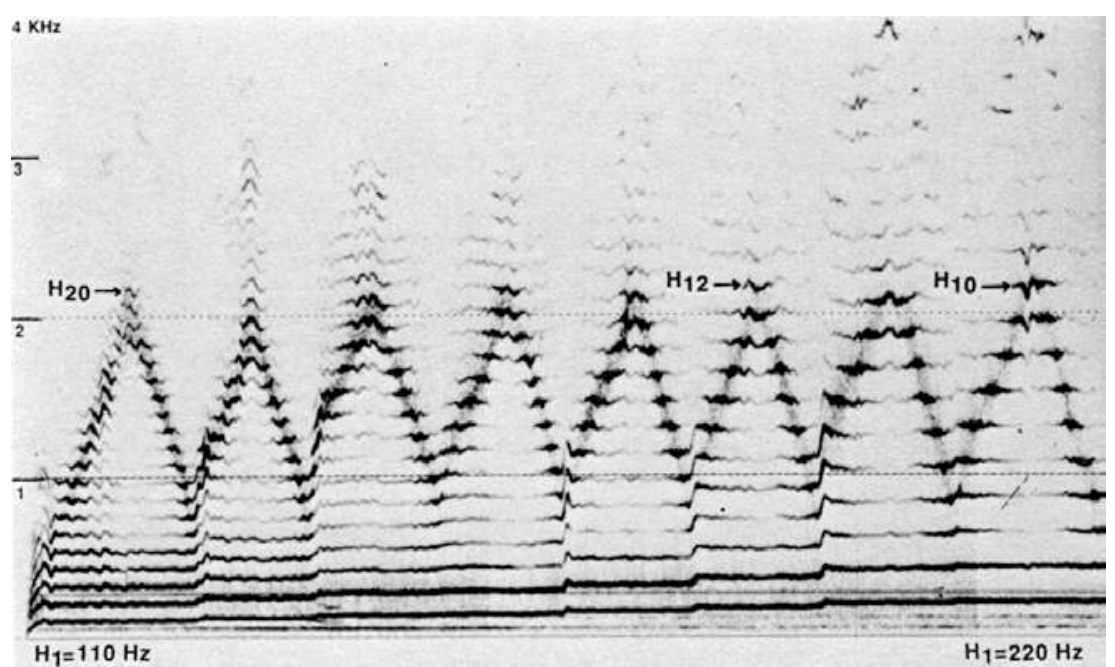

Fig. 2: Échelles d'harmoniques avec différentes hauteurs du fondamental (110 à $220 \mathrm{~Hz}$, selon une échelle diatonique), par Trân Quang Hai. Technique à deux cavités.

Voix nasalisée. H1 et 2 du bourdon très marqués. Les harmoniques bien tracés sur le sonagramme atteignent la limite supérieure de $2200 \mathrm{~Hz}$ avec $\mathrm{H} 10$ dans le cas du fondamental le plus aigu à 220 $\mathrm{Hz}$, et $\mathrm{H} 20$ dans le cas du fondamental le plus grave, à l'octave inférieure $(110 \mathrm{~Hz})$. Les harmoniques exploitables pour une mélodie sont dans le premier cas H4 $(880 \mathrm{~Hz})$ à H10 $(2200 \mathrm{~Hz})$, et dans le deuxième cas H6 $(660 \mathrm{~Hz})$ à H20 $(2200 \mathrm{~Hz})$. 


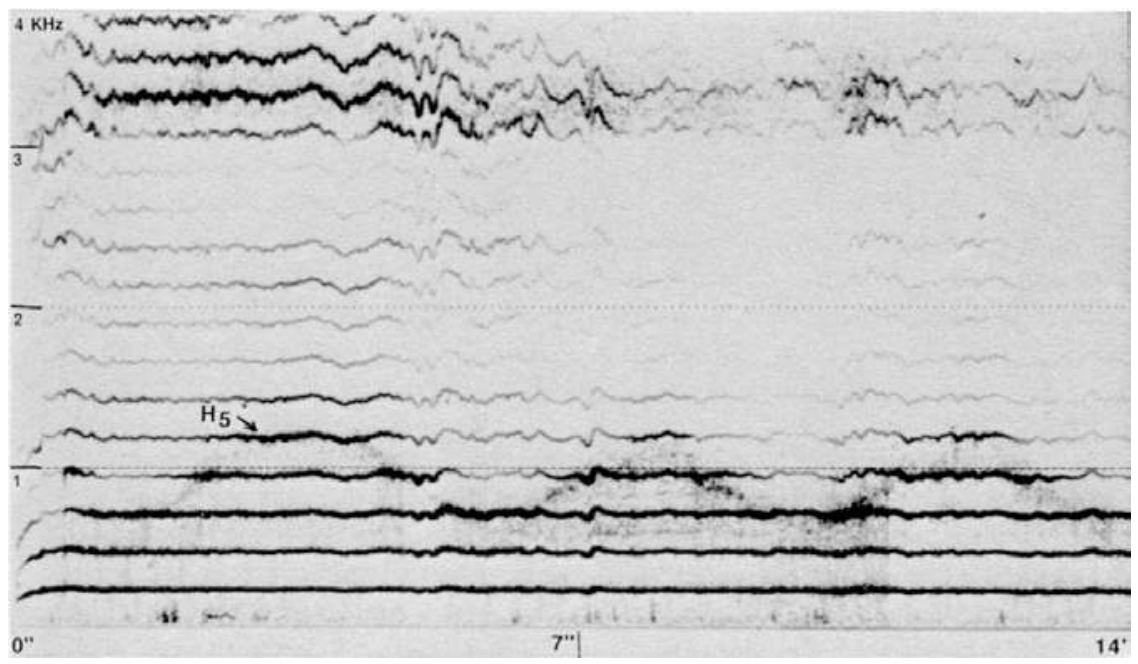

Fig. 3: Échelles d'harmoniques avec bourdon stable, par Minh-Tâm, fille (17 ans) de Trân Quang Hai. Technique à une cavité.

$\mathrm{H} 1(240 \mathrm{~Hz}), 2$ et 3 du bourdon très marqués. Harmoniques utilisables pour une mélodie H3, 4 et 5 $(1200 \mathrm{~Hz})$. Pour une voix de femme, à cause de la hauteur du fondamental plus élevé (ici $240 \mathrm{~Hz}$ ), le nombre d'harmoniques exploitables pour une mélodie est très limité.

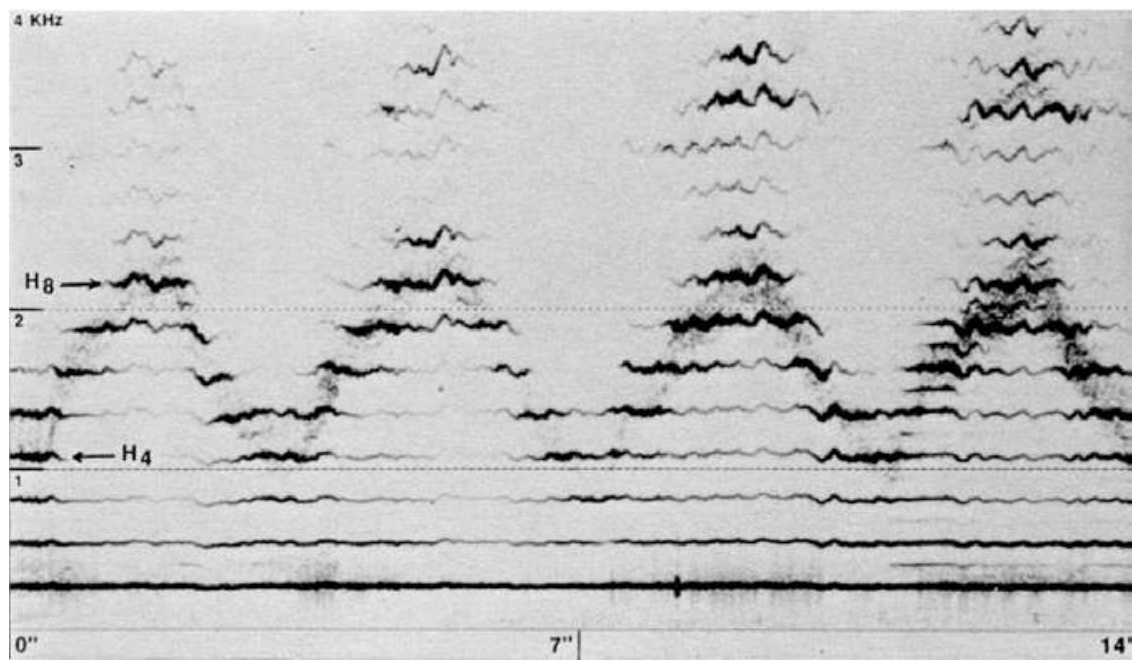

Fig. 4: Échelles d'harmoniques avec bourdon stable, par Minh-Tâm, fille (17 ans) de Trân Quang Hai. Technique à deux cavités.

Voix nasalisée. H1 $(270 \mathrm{~Hz})$. Harmoniques exploitables pour une mélodie H4 $(1080 \mathrm{~Hz})$, 5, 6, 7, 8 $(2160 \mathrm{~Hz})$. H8 fait apparaître H12 et 13 dans la zone aiguë. 


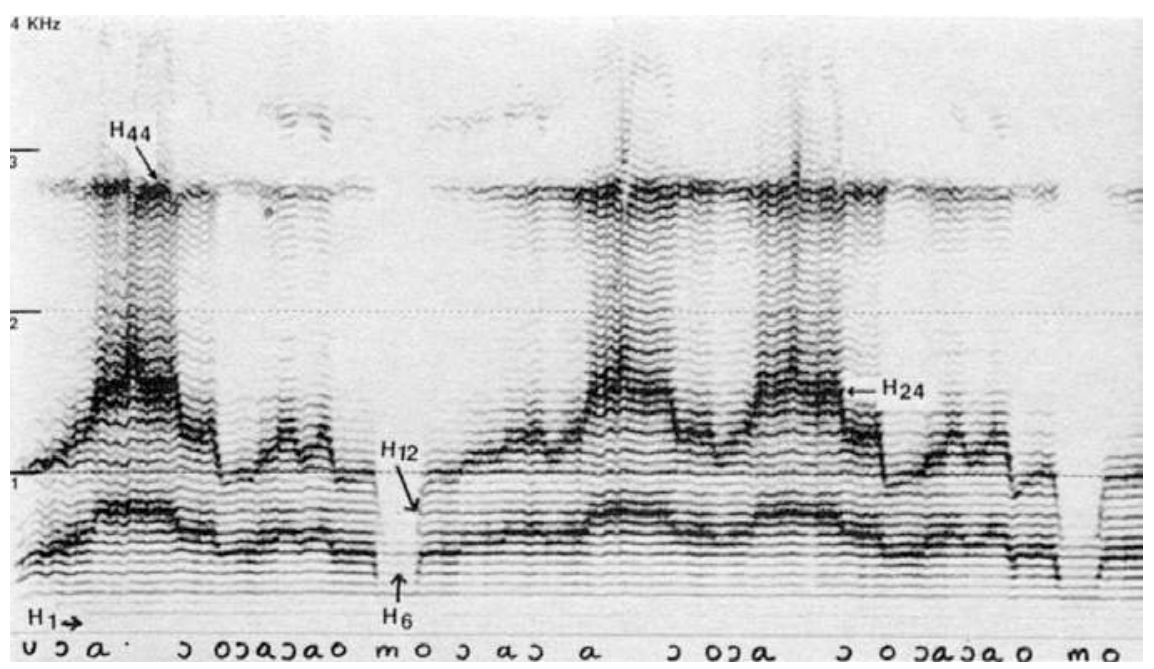

Fig. 5: Tuva (URSS). CD Smithsonian/Folkways, $\mathrm{n}^{\circ} 18$. Style kargiraa, pièce «Artii-sayir» par Vasili Chazir.

Prononciation marquée de voyelles. H1 $(62 \mathrm{~Hz}), 2$ et 3 du bourdon très faibles. Mélodie d'harmoniques H6 $(375 \mathrm{~Hz}), 8,9,10,12(750 \mathrm{~Hz})$. Deuxième zone de mélodie d'harmoniques à l'octave, entre H12 $(750 \mathrm{~Hz})$ et H24 $(1500 \mathrm{~Hz})$. «Bourdon harmonique» aigu H44 $(2690 \mathrm{~Hz})$, plus marqué quand la voyelle a est prononcée (avec une sorte de «colonne grisée» entre 1500 et $2700 \mathrm{~Hz}$. (Transcription musicale de cette pièce dans la notice du CD).

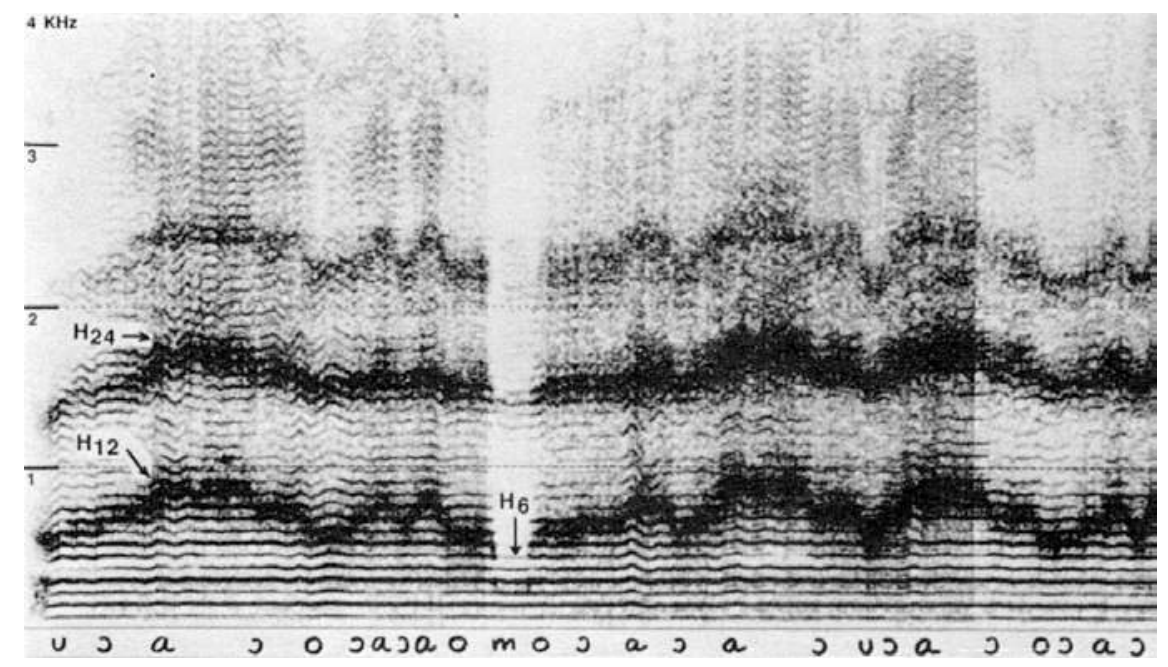

Fig. 6: Imitation par Trân Quang Hai du style kargiraa tuva de la fig. 5.

Technique à une cavité, prononciation marquée de voyelles. Bouche ouverte, sauf quand la mélodie d'harmoniques descend à H6 (alors bouche fermée avec prononciation de la consonne m). Relaxation pharyngienne et abdominale. $\mathrm{H} 1(70 \mathrm{~Hz}), 2,3$ et 4 du bourdon très marqués, $\mathrm{H} 2$ et 4 un peu plus que $\mathrm{H} 1$ et 3. Mélodie d'harmoniques à large bande $\mathrm{H} 6(420 \mathrm{~Hz}), 8,9,10,12(840$ $\mathrm{Hz})$. Deuxième zone de mélodie d'harmoniques à l'octave, entre H18 $(1260 \mathrm{~Hz})$ et H24 $(1680 \mathrm{~Hz})$. Troisième zone de mélodie d'harmoniques très faible, entre 2100 et $2400 \mathrm{~Hz}$. 


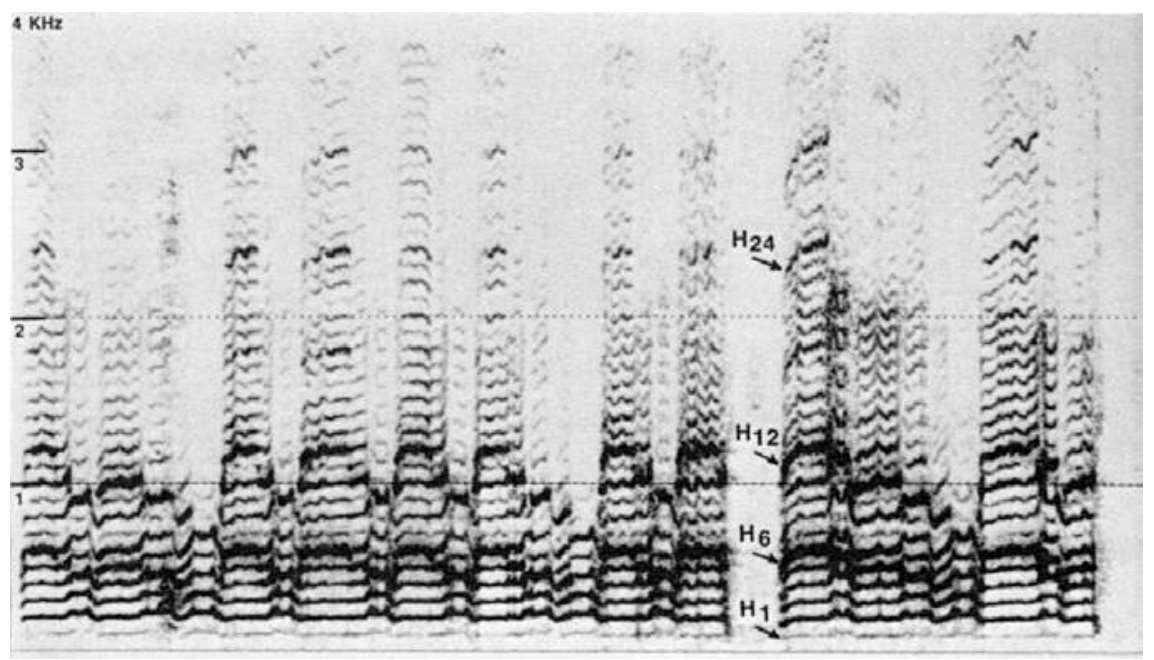

Fig. 7: Xhosa (Afrique du Sud). Enreg. D. Dargie, Phonothèque Musée de l'Homme BM 87.4.1. Style imitant le jeu de l'arc musical umrhubhe, par Nowayilethi.

Alternance de deux fondamentaux à l'intervalle d'un ton. $\mathrm{H} 1(100 \mathrm{~Hz}$ et $110 \mathrm{~Hz})$, très faible; H2, 3 et 4 du bourdon marqués. Mélodie d'harmoniques $\mathrm{H} 4(400 \mathrm{~Hz}), 5,6(600 \mathrm{~Hz})$ quand le fondamental est à $100 \mathrm{~Hz}$; et H4 $(440 \mathrm{~Hz})$ et $\mathrm{H} 5(550 \mathrm{~Hz})$ quand le fondamental est à $110 \mathrm{~Hz}$. Deuxième zone de mélodie d'harmoniques entre 800 et $1200 \mathrm{~Hz}$. Troisième zone plus faible à $2400 \mathrm{~Hz}$; par exemple, lorsque la mélodie d'harmoniques est sur H6, les harmoniques 10 et 12, ainsi que H24 sont très marqués. (Notation musicale dans Dargie 1988: 59)

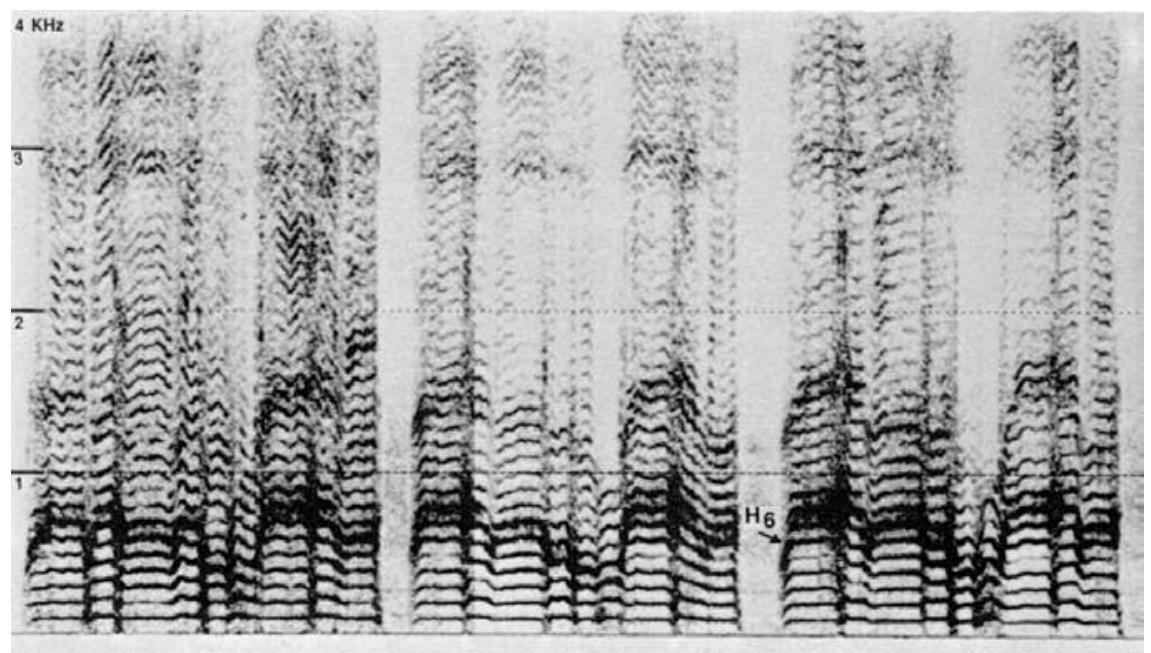

Fig. 8: Imitation par Trân Quang Hai du chant xhosa de la fig. 7.

Technique à une cavité. Voix peu nasalisée, bouche moins ouverte que dans le style kargiraa. Contraction pharyngienne et abdominale. H1 (100 et 110 Hz), 2, 3 et 4 marqués. Mélodie d'harmoniques $\mathrm{H} 4(400 \mathrm{~Hz}), 5,6,8(800 \mathrm{~Hz})$. Technique mal maîtrisée. Absence de deuxième et troisième zone dans l'aigu. 


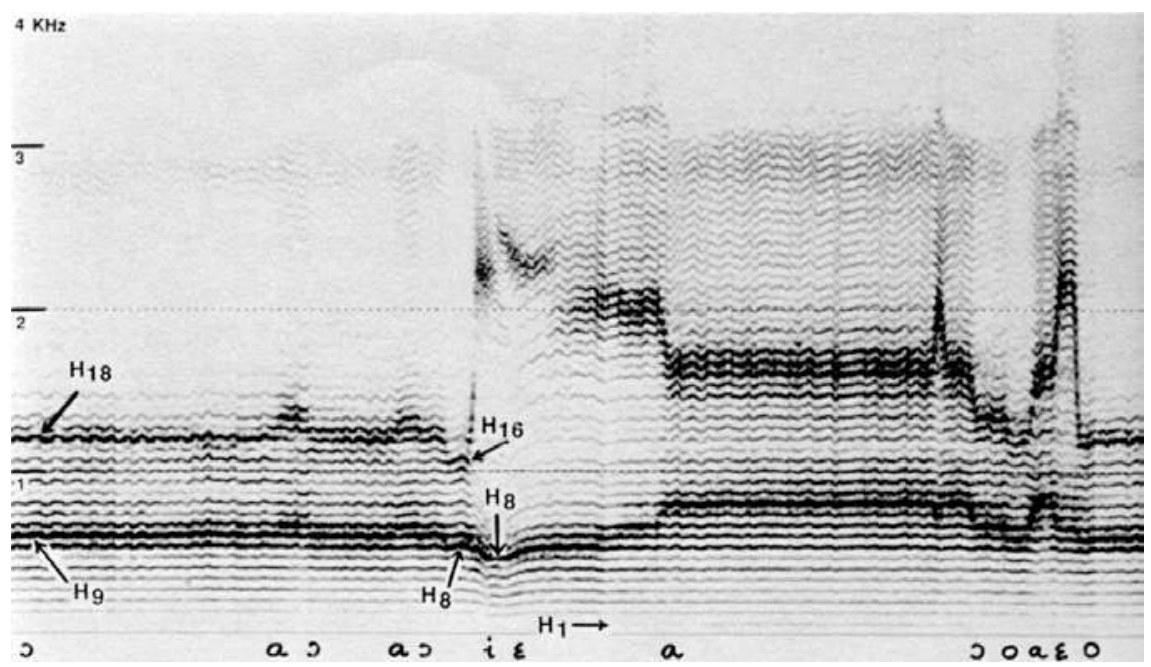

Fig. 9: Tuva (URSS). CD Smithsonian/Folkways, ${ }^{\circ}$ 1. Style «Steppe» kargiraa, par Fedor Tau. Début du chant.

Prononciation marquée de voyelles. H1 $(67 \mathrm{~Hz})$ très faible; H1 est abaissé d'une tierce mineure $(57 \mathrm{~Hz})$ au moment où la voyelle i est prononcée. Paralellement, la mélodie d'harmoniques baisse également d'une tierce mineure, l'harmonique restant le même (H8). Mélodie d'harmoniques à large bande $\mathrm{H} 8(536 \mathrm{~Hz})$ et $456 \mathrm{~Hz}), 9,10,12(804 \mathrm{~Hz})$. Deuxième zone de mélodie d'harmoniques entre $\mathrm{H} 16(1072 \mathrm{~Hz})$ et $\mathrm{H} 30(2010 \mathrm{~Hz})$. Pour les voyelles postérieures $\underline{\underline{o}}$, $\underline{\underline{a}}, \underline{\mathrm{a}}$, la deuxième zone de mélodie d'harmoniques est à l'octave $\mathrm{H} 8-\mathrm{H} 16, \mathrm{H} 9-\mathrm{H} 18, \mathrm{H} 10-\mathrm{H} 20, \mathrm{H} 12-\mathrm{H} 24$.

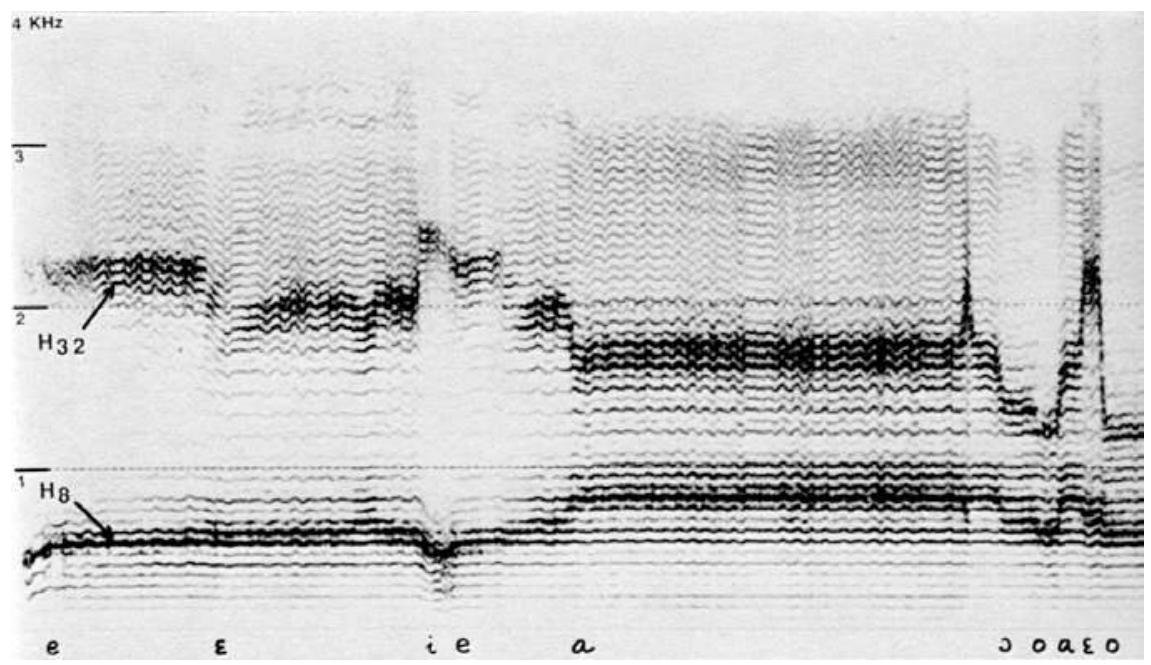

Fig. 10: Reprise du chant de fig. 9. Même mélodie d'harmoniques, mais différence de la deuxième zone en raison de la différence des voyelles. Pour les voyelles antérieures $\underline{i}, \underline{e}, \underline{\varepsilon}$, la deuxième zone de mélodie d'harmoniques est plus haute la voyelle e au début de la reprise (fig. 10) H8 - H32 (= double octave); la voyelle i H9 - large bande de H28 à 30 . 


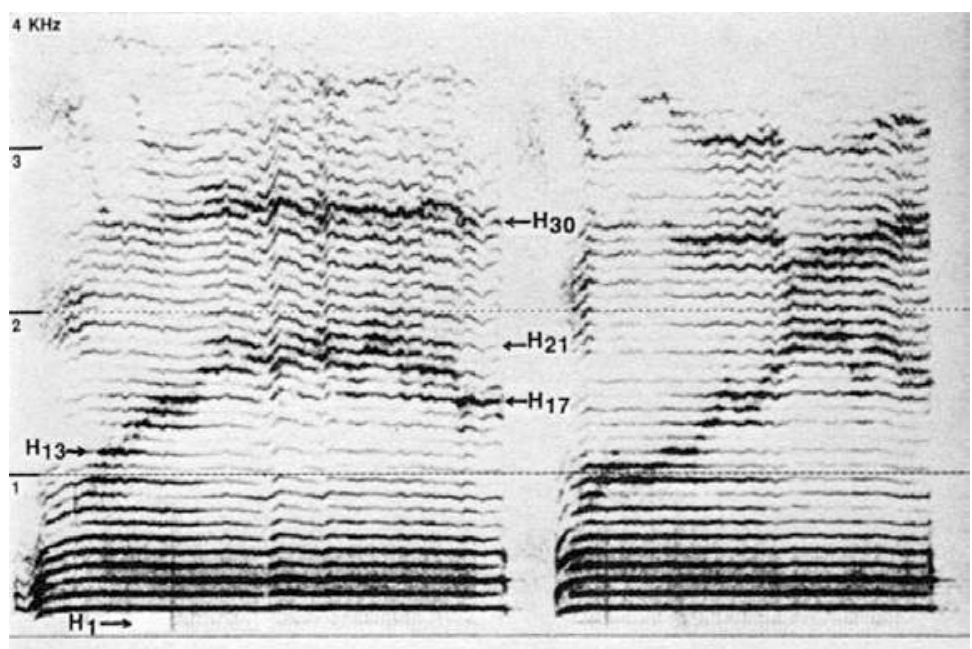

Fig. 11: Mongolie. Film Le chant des harmoniques. Style khargiraa khöömii, démonstration pour l'interview, par T. Ganbold.

$\mathrm{H} 1(85 \mathrm{~Hz})$ très faible; $\mathrm{H2}, 3,4,5$ et 6 du bourdon très marqués. Mélodie d'harmoniques faible, avec des harmoniques impairs H13 (1105 Hz), 15, 17, 19, 21 (1785 Hz). Dans l'aigu, une zone d'harmoniques faible entre $\mathrm{H} 28$ et $\mathrm{H} 30(2630$ à $2800 \mathrm{~Hz})$.

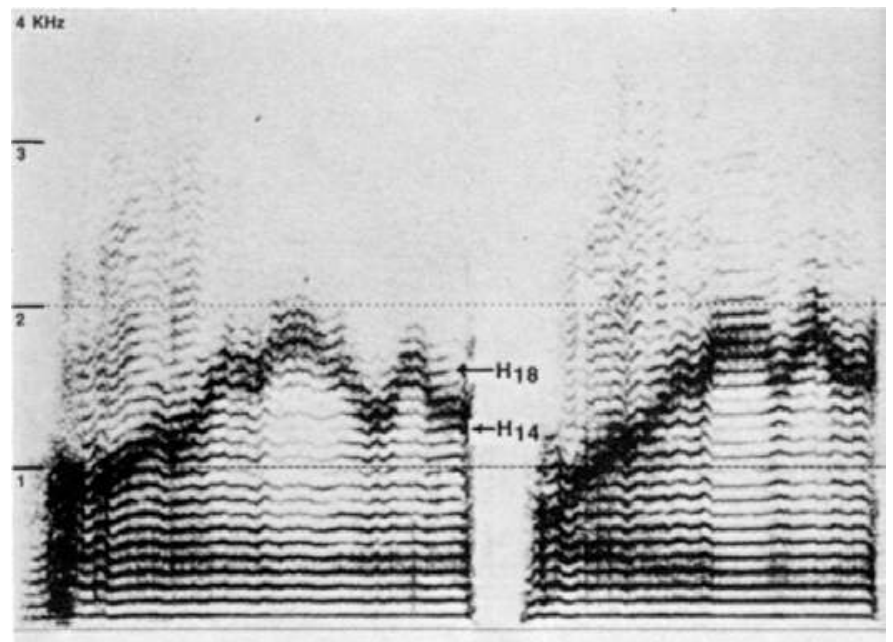

Fig. 12: Imitation par Trân Quang Hai du style khargiraa khöömii mongol de la fig. 11.

Technique à deux cavités. H1 $(90 \mathrm{~Hz})$ de même que H2, 3, 4 et 5 très marqués. Mélodie d'harmoniques avec des harmoniques paires H12 $(1080 \mathrm{~Hz}), 14,16,18,20(1800 \mathrm{~Hz})$. Relaxation pharyngiennee et abdominale. 


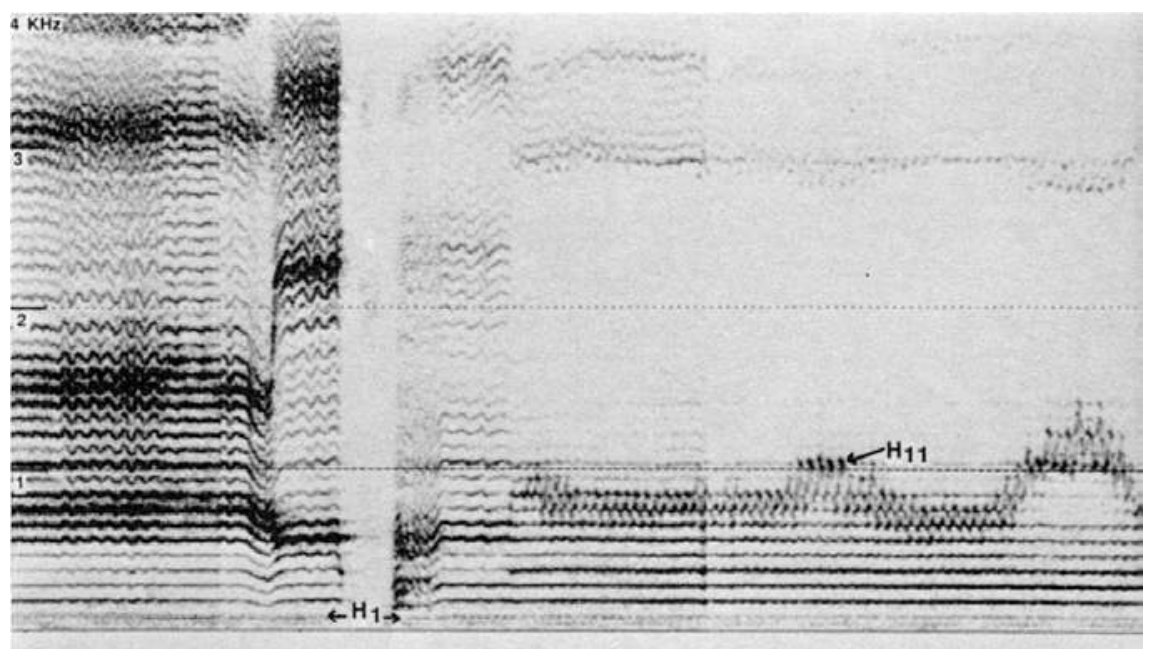

Fig. 13: Tuva (URSS). CD Smithsonian/Folkways, $\mathrm{n}^{\circ}$ 14. Pièce intitulée borbannadir, par Anatolii Kuular.

Manifestement les styles kargiraa et borbannadir se succèdent. Tracé caractéristique de kargiraa, avec vibrato six battements/seconde en forme d'ondulations. H1 $(95 \mathrm{~Hz})$ faible. Dans la partie borbannadir, H1 (95 Hz) faible; H2, 3 et 4 marqués. Pulsation à huit battements/seconde «en zigzag» sur la mélodie d'harmoniques à bande large H7 $(685 \mathrm{HZ}), 8,9$ et $11(1045 \mathrm{~Hz})$.

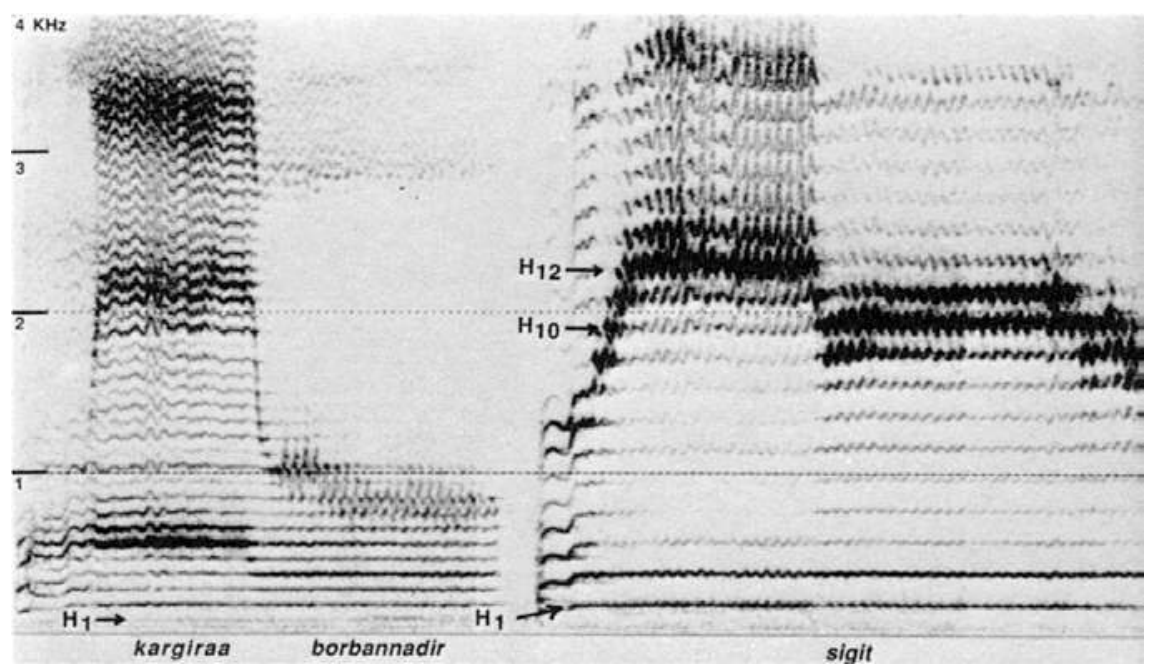

Fig. 14: Même pièce que fig. 13, un peu plus loin.

Manifestement trois styles. Dans le premier segment, styles kargiraa et borbannadir enchainés, avec H1 $(95 \mathrm{~Hz})$ faible. Après une courte interruption, deuxième segment de style différent, avec un saut d'octave du fondamental, $\mathrm{H} 1(190 \mathrm{~Hz})$ et $\mathrm{H} 2$ bien marqués. Mélodie d'harmoniques à bande large, avec pulsation à sept battements/seconde «en zigzag», H9 $(1710 \mathrm{~Hz}), 10$ et $12(2280 \mathrm{~Hz})$. 


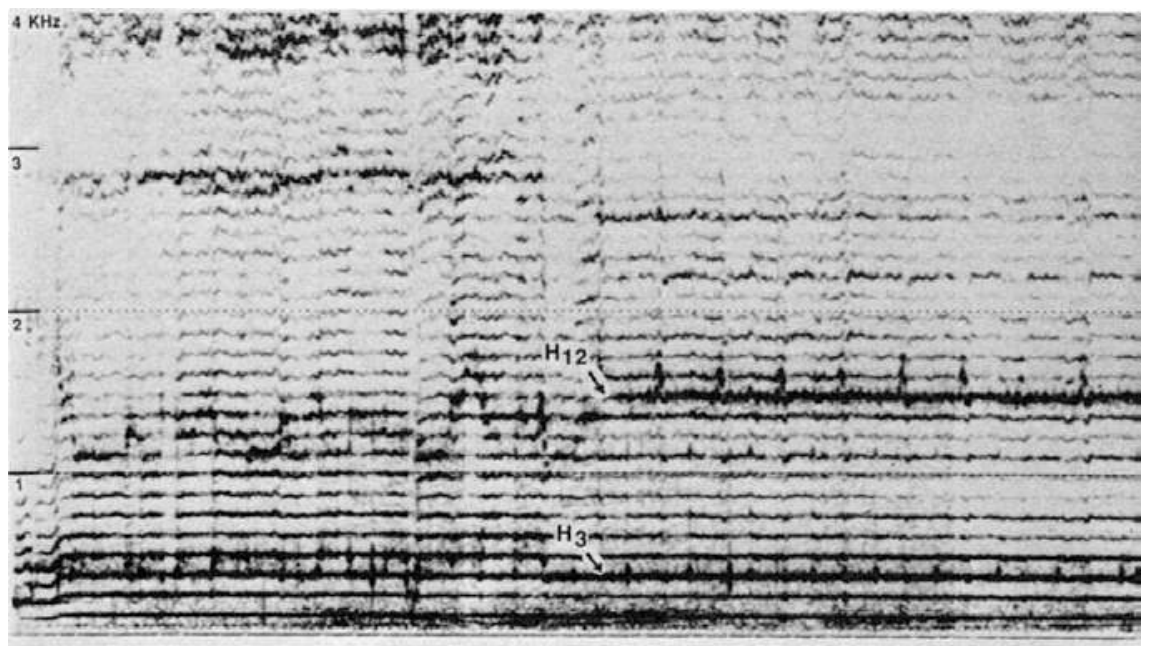

Fig. 15: Tuva (URSS). Disque Melodia, face A, plage 5. Style borbannadir, pièce «Boratmoj, Spoju Borban», par Oorzak Xunastar-ool.

H1 $(120 \mathrm{~Hz}$ ) et 2 marqués. H3 du bourdon très marqué (on entend bien ce deuxième bourdon à la quinte). Mélodie d'harmoniques H8 $(980 \mathrm{~Hz}), 9,10$ et $12(1440 \mathrm{~Hz})$. Accents rythmiques visibles surtout sur H3, 12 et 13 .

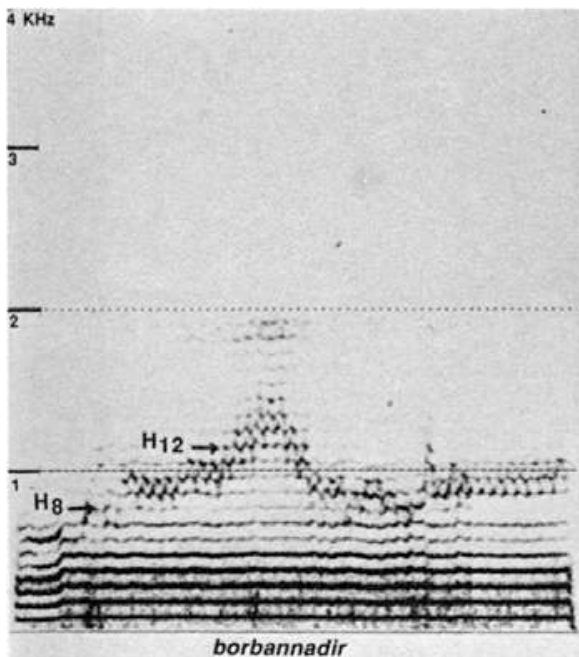

Fig. 16: Imitation par Trân Quang Hai du style borbannadir de la fig. 13.

Technique à une cavité. Émission nasalisée, bouche presque fermée, prononciation de la voyelle o. H1 $(95 \mathrm{~Hz}), 2$ à 5 très marqués. Mouvements rapides de la langue en avant et en arrière pulsation «en zigzag» sur la mélodie d'harmoniques à bande large H9 $(855 \mathrm{~Hz})$ à $13(1235 \mathrm{~Hz})$.

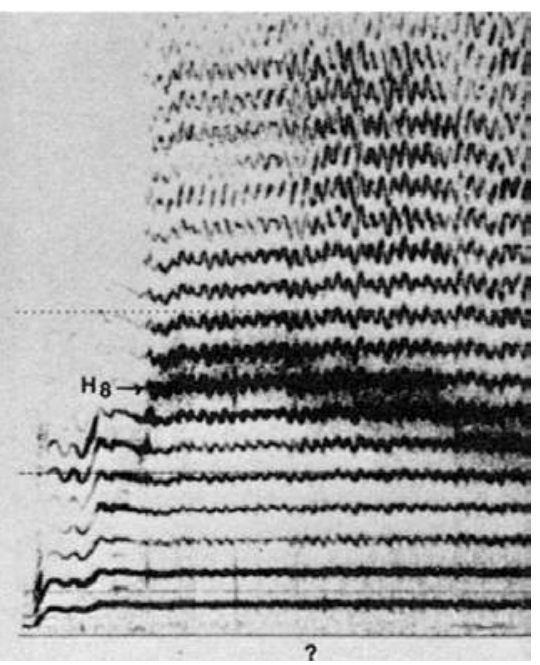

Fig. 17: Imitation par Trân Quang Hai du style non identifié de la fig. 14.

Technique à 2 cavités. Forte nasalisation, contraction pharyngienne et abdominale. $\mathrm{H} 1(190 \mathrm{~Hz})$ et 2 très marqués. Rapides aplatissements de la pointe de la langue contre le palais, et simultanément tremblement de la mâchoire inférieure pulsation «en zigzag» sur la mélodie d'harmoniques à bande large H6 $(1140 \mathrm{~Hz})$ et $8(1520 \mathrm{~Hz})$. 


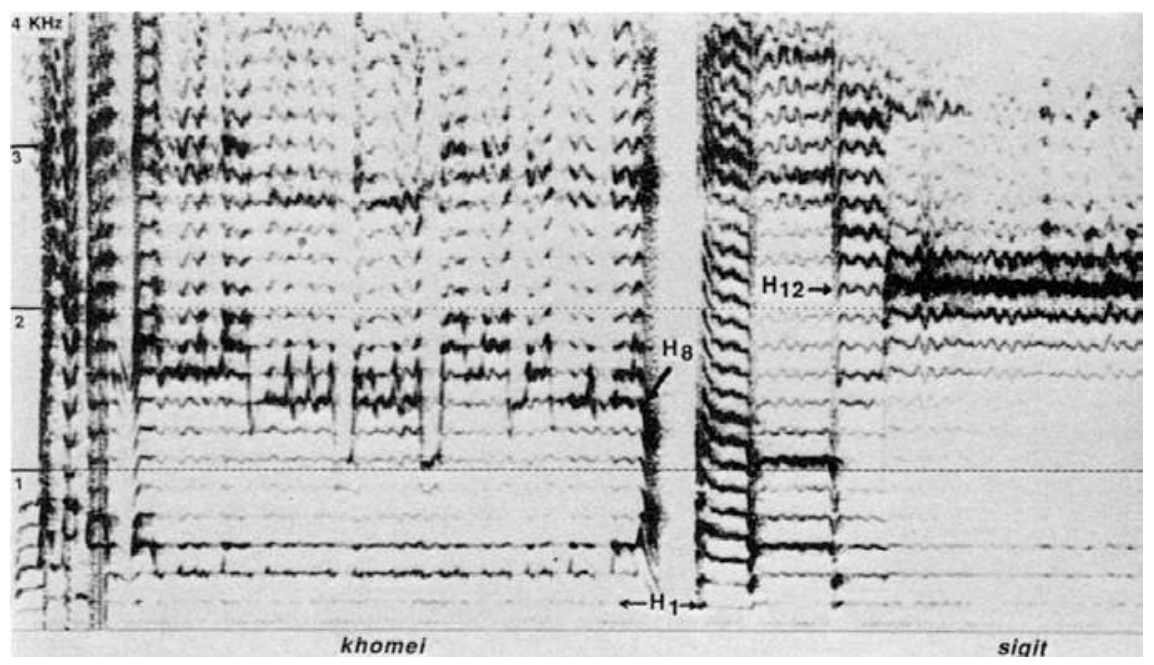

Fig. 18: Tuva (URSS). CD Smithsonian/Folkways, $n^{\circ}$ 8. Styles khoomei et sigit, par Tumat Kara-ool. H1 $(175 \mathrm{~Hz})$. Pour le khoomei, H1 faible; H2 et 3 marqués. Mélodie d'harmoniques à bande large H6 $(1050 \mathrm{~Hz}), 8,9,10(1750 \mathrm{~Hz})$. Pulsation rythmique visible sous forme de traits verticaux dirigés alternativement vers le haut et le bas à partir de la ligne horizontale indiquant la mélodie d'harmoniques. Pour le sigit (cf. aussi fig. 21 et 22), H1, 2 et 3 du bourdon faible. Mélodie d'harmoniques H12 (2050 $\mathrm{Hz}$ ) et ornement $\mathrm{H} 13$; les autres harmoniques n'apparaissant pas sur l'extrait de ce sonagramme.

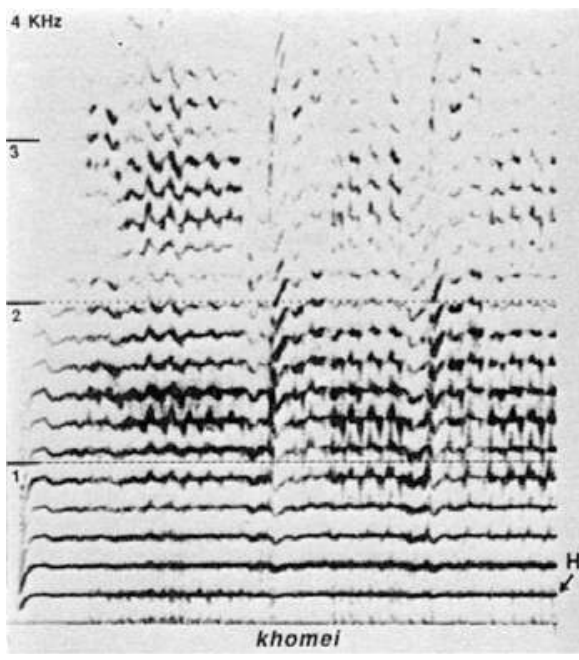

Fig. 19: Imitation par Trân Quang Hai du style khoomei tuva de la fig. 18.

Technique à deux cavités. Voix peu nasalisée. Contraction pharyngienne modérée. Bouche moins ouverte que pour le sigit. Mouvements rythmiques de la lèvre inférieure vers la lèvre supérieure: pulsation rythmique visible sous forme de traits verticaux alternativement audessus et au-dessous de la ligne horizontale.

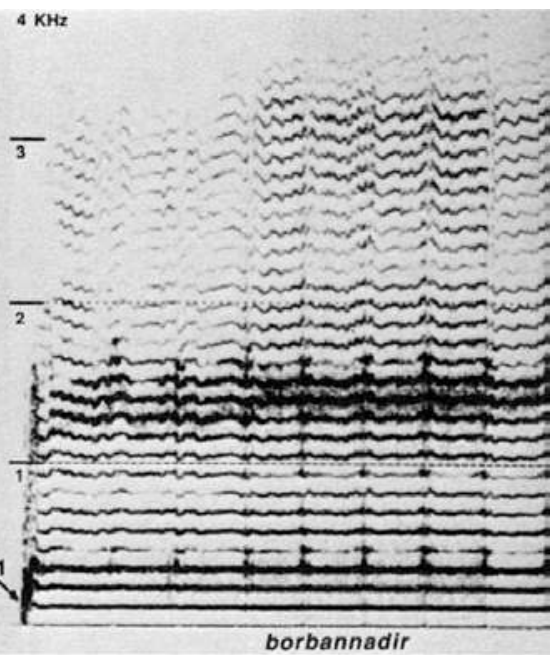

Fig. 20: Imitation par Trân Quang Hai du style borbannadir tuva de la fig. 15 .

Technique à deux cavités. Voix nasalisée. Contraction pharyngienne modérée comme pour la fig. 18a, mais la bouche encore moins ouverte, arrondie. Coups de langue rythmiques contre le palais, bien marqués sur $\mathrm{H} 3 \mathrm{du}$ bourdon et sur les harmoniques supérieurs. H1 $(175 \mathrm{~Hz}), 2$ et 3 très marqués; mélodie d'harmoniques H10 $(1750 \mathrm{~Hz})$ et H $12(2050 \mathrm{~Hz})$. 


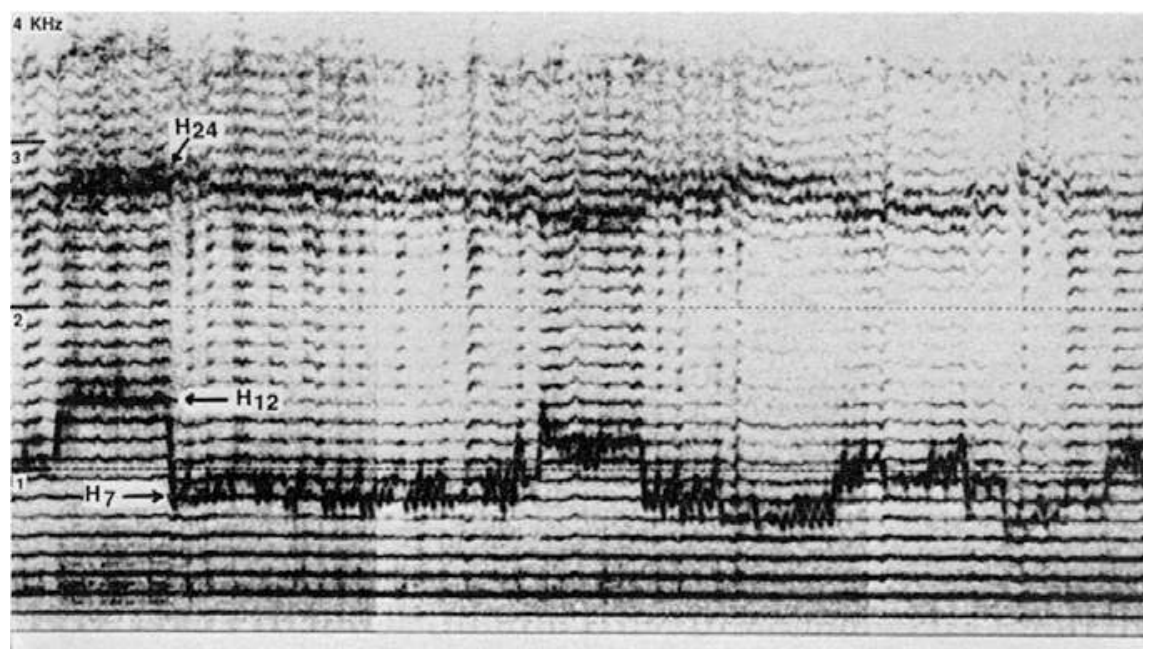

Fig. 21: Tuva (URSS). CD Smithsonian/Folkways, $n^{\circ}$ 15. Style ezengileer, par Marzhimal Ondar. H1 $(120 \mathrm{~Hz}), 2,3,4$ du bourdon très marqués. Mélodie d'harmoniques H6 $(720$ Hz), 7, 8, 9, 10, 12 $(1440 \mathrm{~Hz})$. Le tracé est caractérisé par des accents rythmiques «en zigzag» sur 2 ou 3 harmoniques (l'extrait choisi ne comporte pas le frappement de doigts sur un bol de thé, indiqué sur la notice du CD). Dans l'aigu, une deuxième zone d'harmoniques entre H22 et H24 (2880 Hz).

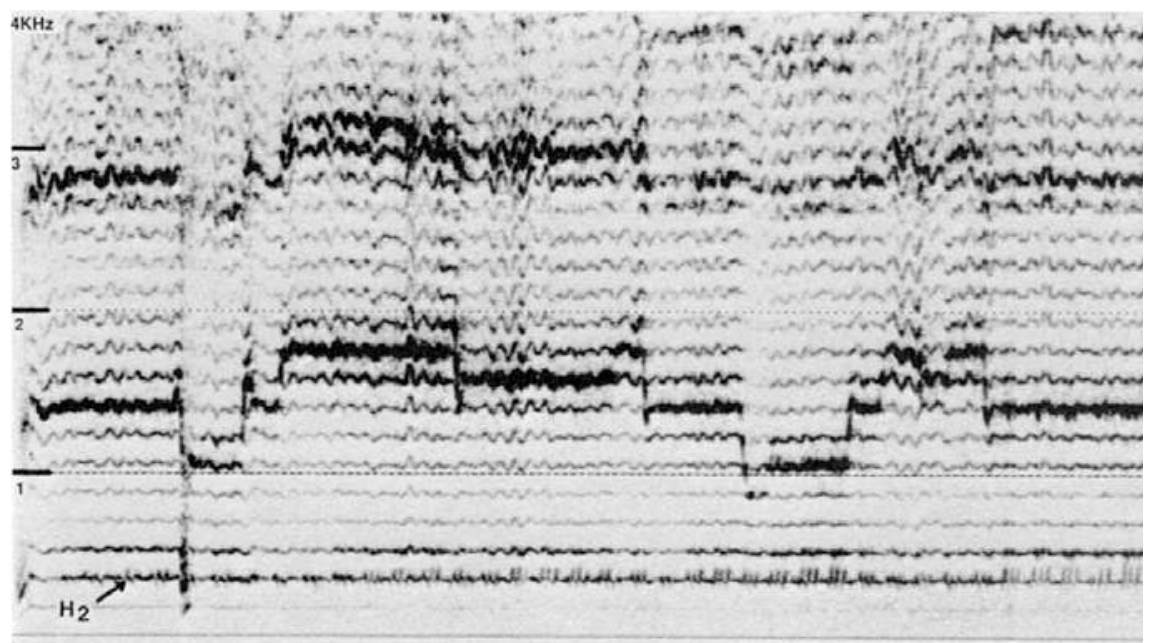

Fig. 22: Tuva (URSS). CD Smithsonian/Folkways, nº 13. Pièce intitulée «Borbannadir with finger strokes», par Tumat Kara-ool.

H1 $(180 \mathrm{~Hz})$ faible. «Hachures» irrégulieres sur H2, rythmiquement différentes du vibrato en forme d'ondulations marqué sur les harmoniques supérieurs. Mélodie d'harmoniques H6 (1080), 8, 9, $10(1800 \mathrm{~Hz})$. Deuxième zone de mélodie d'harmoniques à l'octave, sauf H10 — H18. 


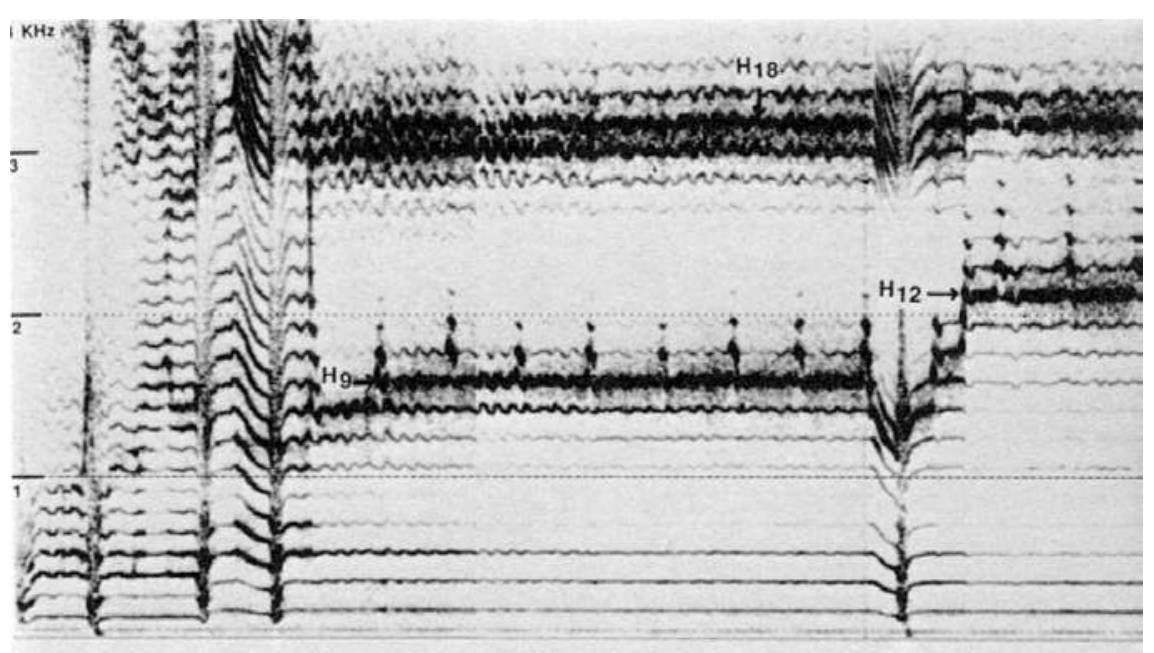

Fig. 23: Tuva (URSS). CD Smithsonian/Folkways, $\mathrm{n}^{\circ}$ 3. Style sigit, pièce «Alash» par Mergen Mongush.

Début chanté avec des paroles en voix naturelle. Abaissement momentané du fondamental à une tierce mineure. H1 $(190 \mathrm{~Hz}), 2$ et 3 du bourdon marqués. Mélodie d'harmoniques H8 $(1520 \mathrm{~Hz}), 9$, 10 et 12 ; ornements rythmiques sur H10 et 13 . Dans l'aigu, «bourdon harmonique» à l'octave de la mélodie d'harmoniques $(\mathrm{H} 9-\mathrm{H} 18)$ et à la quinte $(\mathrm{H} 12-\mathrm{H} 18)$. Zone de rejection d'harmoniques entre le bourdon (H1 à H3) et la mélodie d'harmoniques, et entre celle-ci et le bourdon aigu (H18).

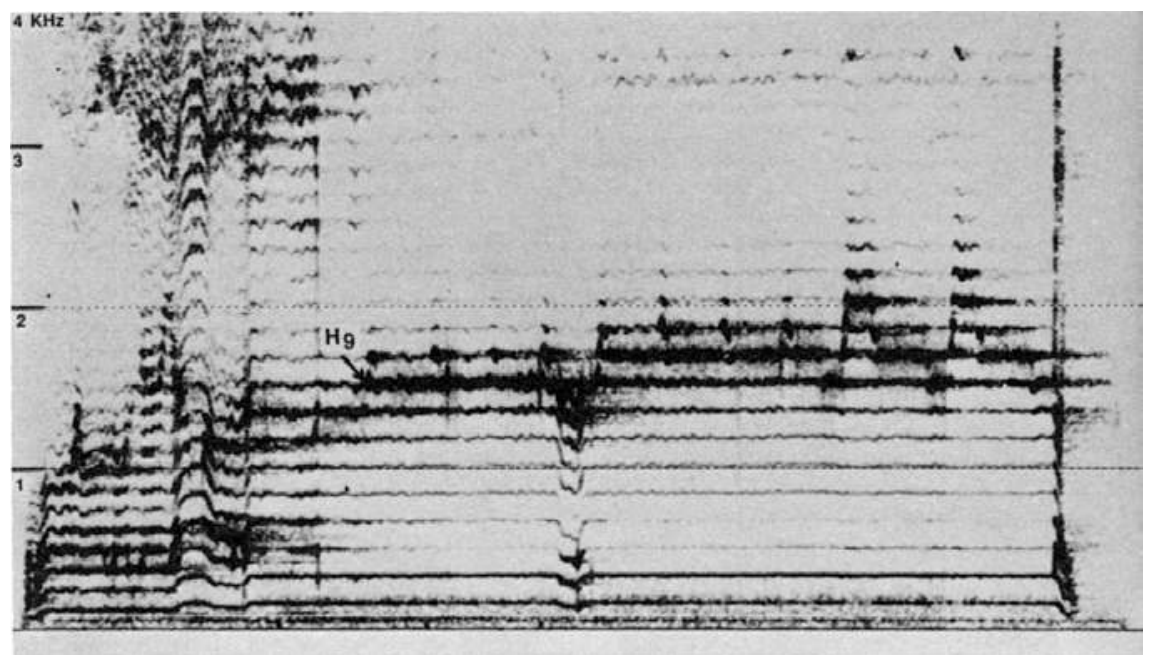

Fig. 24: Tuva (URSS). Disque Melodia, face A, plage 1. Style sigit, pièce «Reka Alas» (même pièce que fig. 23).

Début chanté avec des paroles en voix naturelle. Abaissement momentané du fondamental d'une tierce mineure. H1 $(170 \mathrm{~Hz})$ et 2 marqués. Mélodie d'harmoniques H9 $(1530 \mathrm{~Hz}), 10,12(2030 \mathrm{~Hz})$; ornements rythmques sur $\mathrm{H} 10$ et 11 . Absence de deuxième zone dans l'aigu. 


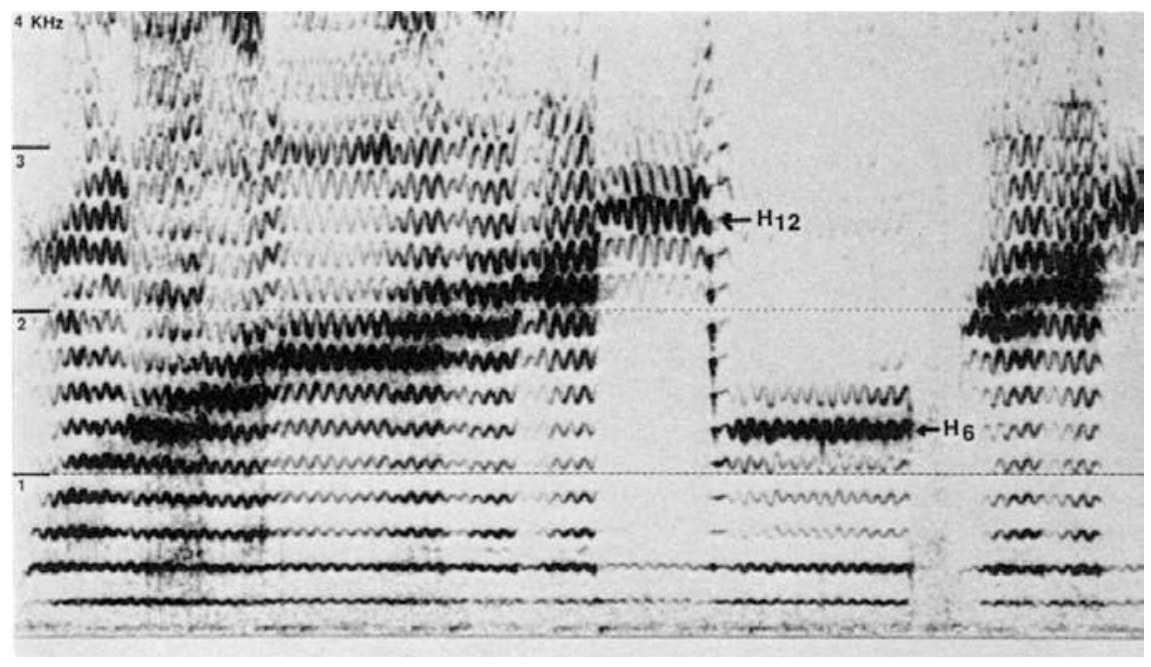

Fig. 25: Mongolie. Disque Tangent, face B, plage 1. Pièce intitulé «Mouth music», par Sundui (cf. aussi le film Le chant des harmoniques). Style non nommé sur la notice du disque, mais sans doute «khöömi de ventre», le style préféré de Sundui.

H1 $(210 \mathrm{~Hz})$ et 2 marqués, sauf quand la mélodie d'harmoniques est sur H12. Mélodie d'harmoniques à bande large $\mathrm{H} 5(1050 \mathrm{~Hz}), 6,7,8,9,10$ et $12(2510 \mathrm{~Hz})$. Vibrato en forme d'ondulations.

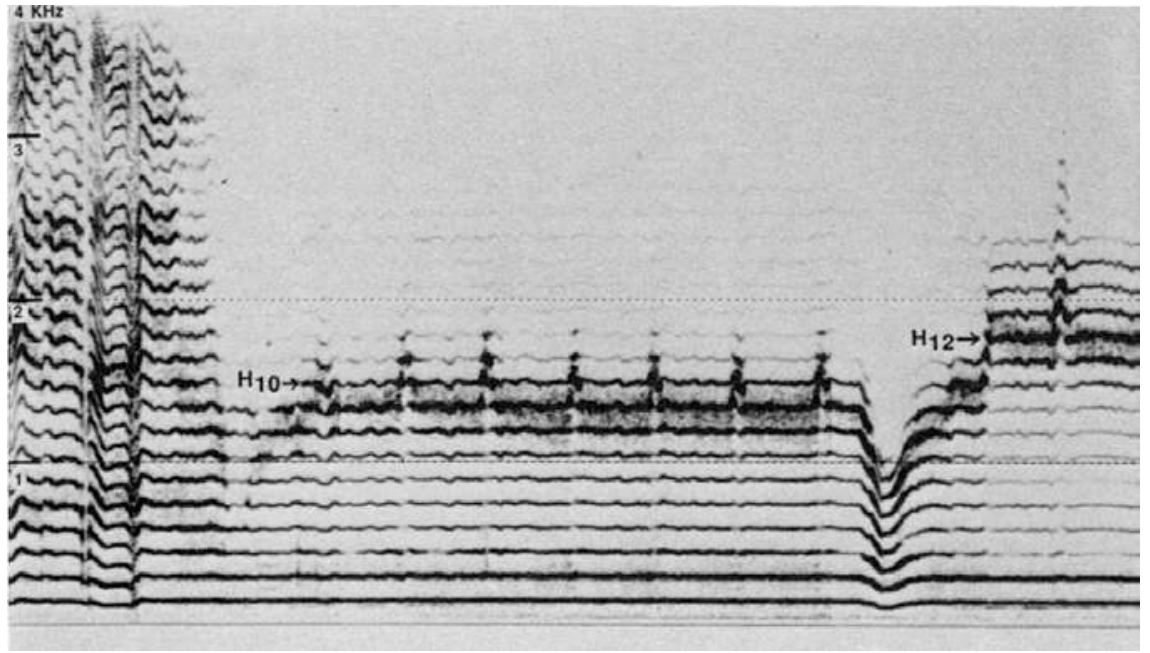

Fig. 26: Imitation par Trân Quang Hai du style sigit tuva de la fig. 23.

Technique à deux cavités. Forte nasalisation. Contraction abdominale et pharyngienne. Rétrécissement de la colonne d'air. Pour obtenir les ornements rythmiques (H10 à partir de la ligne mélodique H9, et H13 à partir de la ligne mélodique H12), la pointe de la langue est légèrement aplatie contre le palais. H1 $(145 \mathrm{~Hz})$ et H2 très marqués. Même mélodie d'harmoniques que fig. 23 et 24. Absence de deuxième zone dans l'aigu, comme fig. 24. 


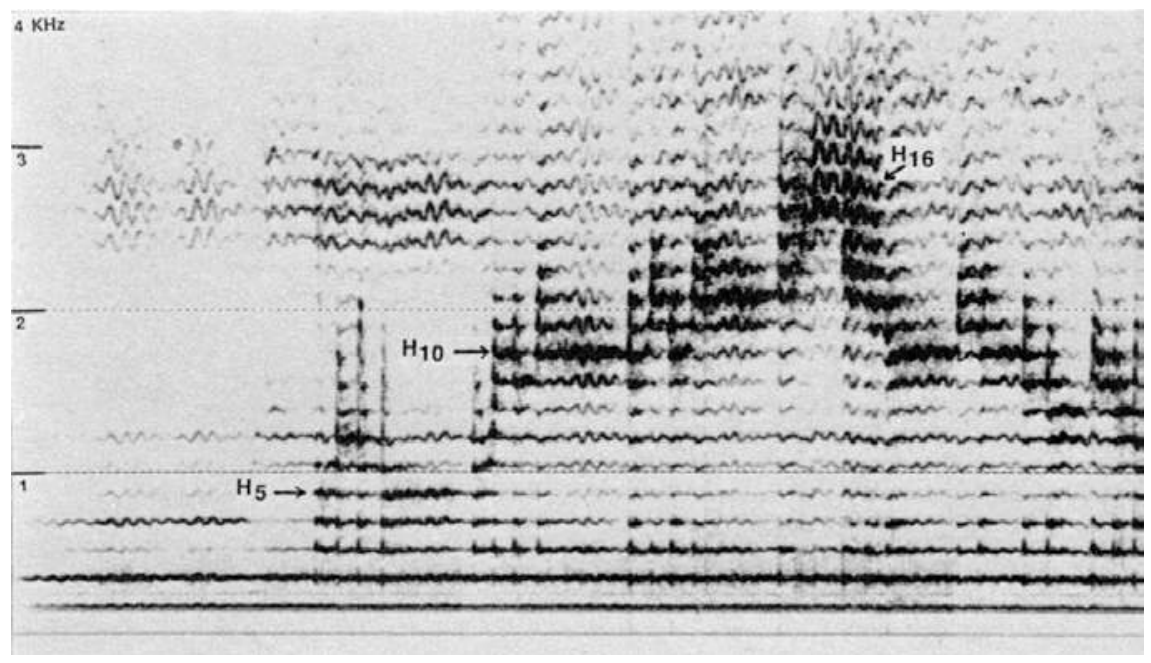

Fig. 27: Rajasthan. Enregistrement J. Levy. Phonothèque du Musée de l'Homme BM78.2.1.

$\mathrm{H} 1(170 \mathrm{~Hz})$ et 2 très marqués. Mélodie d'harmoniques H5 $(850 \mathrm{~Hz}), 6,8,9,10,11,12,13,16(2720$ $\mathrm{Hz}$ ), à large bande. Accents rythmiques, sans doute pour imiter le jeu de la guimbarde. Plus grand ambitus de toutes les pièces analysées dans cet article.

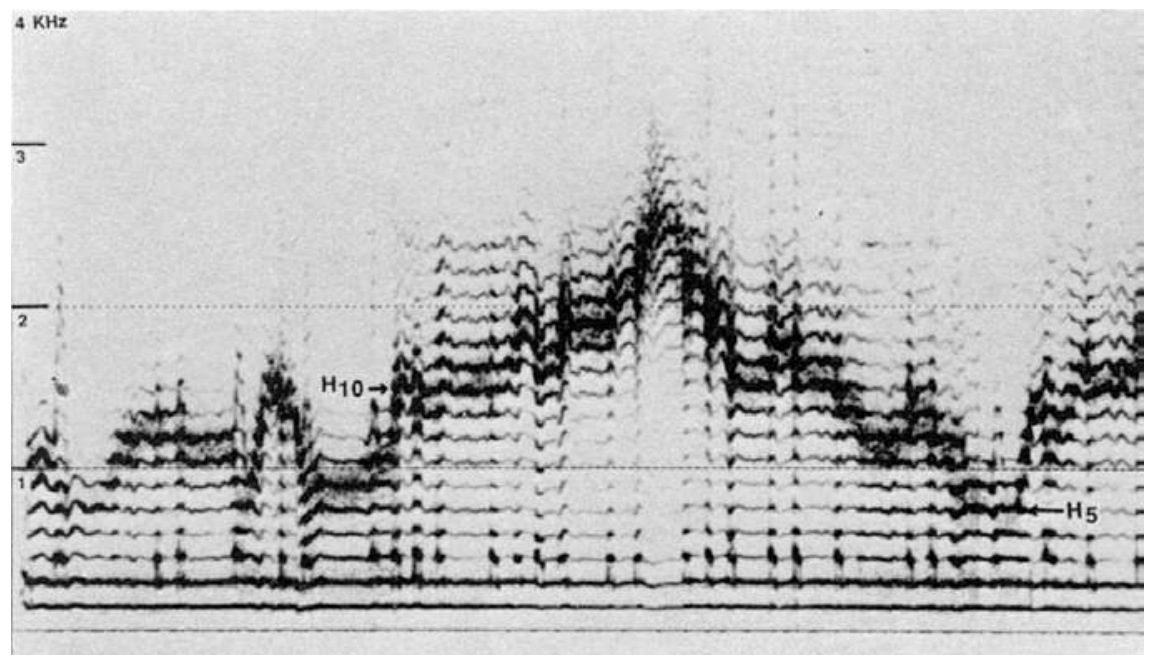

Fig. 28: Imitation par Trân Quang Hai du chant du Rajasthan de la fig. 27.

Technique à deux cavités. Voix fortement nasalisée. Forte contraction pharyngienne et abdominale (comme pour le sigit). Coups de langue rythmiques contre le palais, ce qui donne sur le tracé des lignes verticales comme sur la fig. 27. H1 (150 Hz), H5 (750 Hz) à H $16(2400 \mathrm{~Hz})$. 


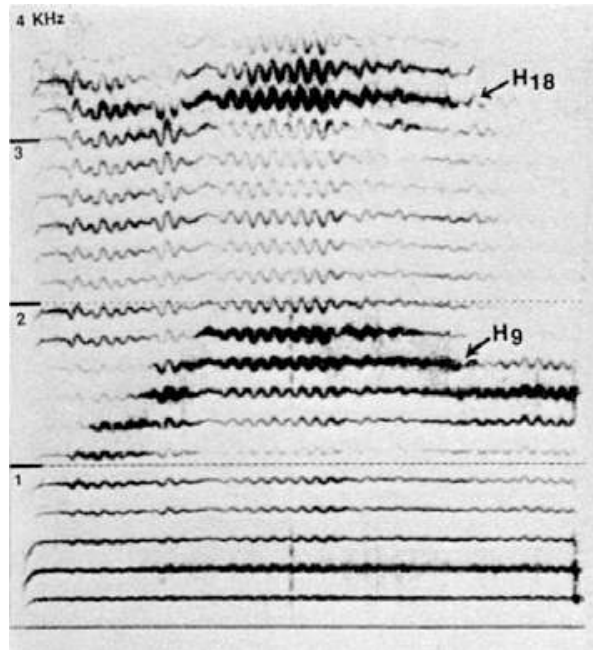

Fig. 29: Mongolie. Probablement style tzeedznii khöömii («khöömii de poitrine»), appelé dans le film Le chant des harmoniques par erreur bagalzuuriin khöömi, («khöömi de gorge»). Démonstration pour l'interview, par T. Ganbold.

H1 $(180 \mathrm{~Hz})$ marqué, H2 très marqué. Mélodie d'harmoniques H8 (1440 Hz), 9, 10 (1800 Hz). Dans l'aigu, $2^{\mathrm{e}}$ zone à l'octave (H18 à $3240 \mathrm{~Hz}$ ).

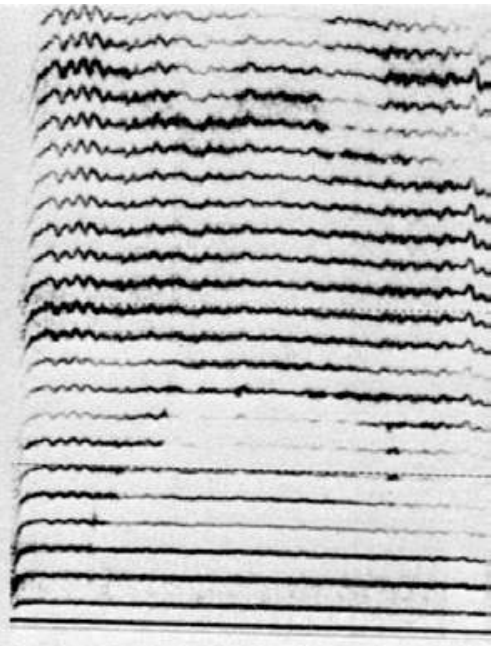

Fig. 30: Même chanteur. Style khamryn khöömii («khöömii de nez»).

H1 $(170 \mathrm{~Hz}), 2$ et 3 marqués. Mélodie d'harmoniques très faible $\mathrm{H} 8,9$ et 10 , «noyée» dans les harmoniques couvrant toute l'étendue du spectre.

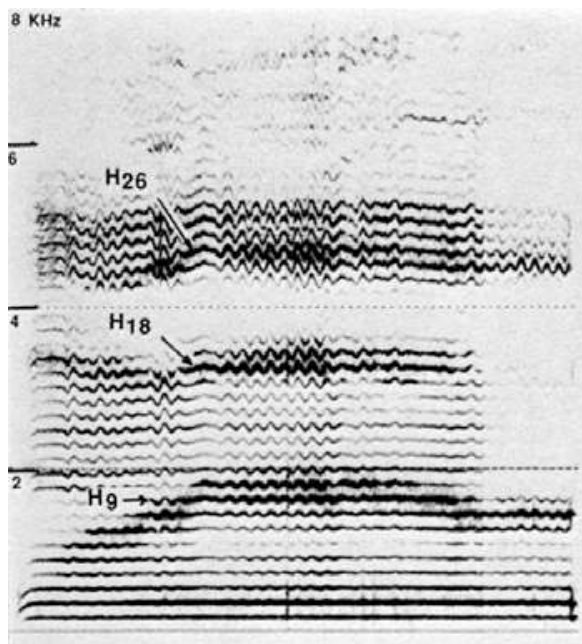

Fig. 31: Même signal que fig. 29.

Analyse à $8 \mathrm{KHz}$, montrant une troisième zone à large bande au dessus de $4000 \mathrm{~Hz}$.

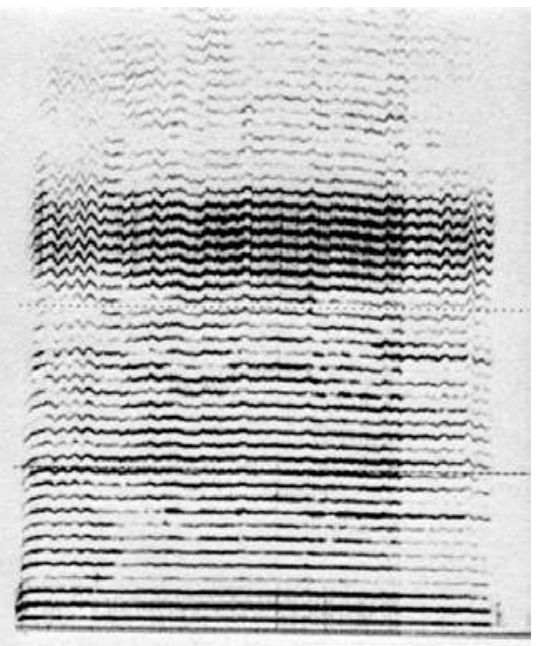

Fig. 32: Même signal que fig. 30.

Analyse à $8 \mathrm{KHz}$, montrant une troisième zone à large bande au dessus de $4000 \mathrm{~Hz}$. 


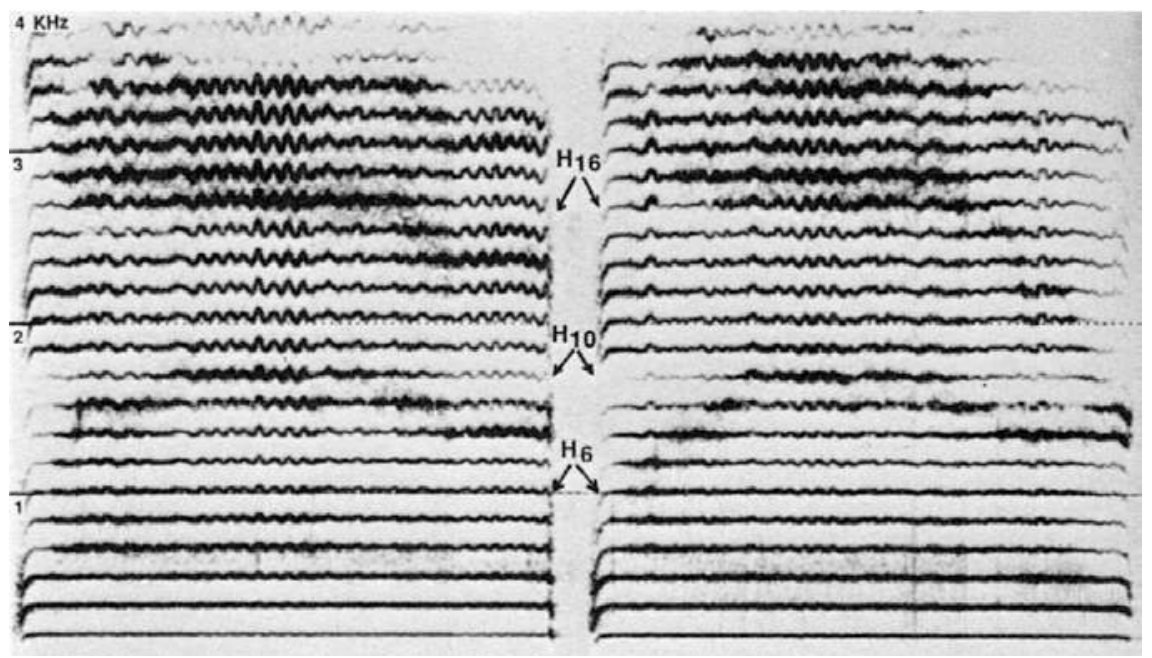

Fig. 33: Mongolie. Probablement style bagalzuuliin khöömii, («khöömii de gorge»), appelé dans le film Le chant des harmoniques par erreur tseedznii khöömi («khöömi de poitrine»). Démonstration pour l'interview, par T. Ganbold.

Bouche grande ouverte. H1 $(170 \mathrm{~Hz}), 2,3,5,6$ du bourdon très marqués. Mélodie d'harmoniques H8 $(1360 \mathrm{~Hz}), 9,10(1700 \mathrm{~Hz})$. Dans l'aigu, une deuxième zone d'harmoniques à bande large H16 à $20(2720$ à $3400 \mathrm{~Hz}$ ). Une analyse à $8000 \mathrm{~Hz}$ (non reproduite ici) montre une troisième zone très marquée au-dessus de $4000 \mathrm{~Hz}$.

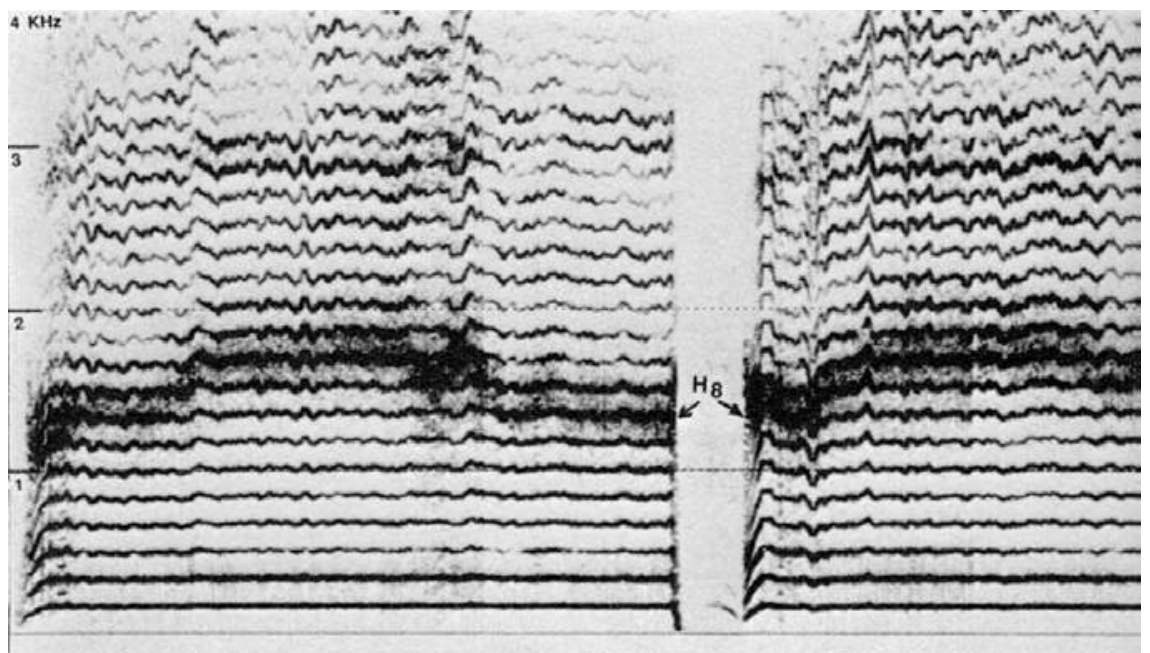

Fig. 34: Imitation par Trân Quang Hai du style de la fig. 33.

Technique à deux cavités avec l'arrière de la langue mordue par les molaires. Contraction pharyngienne avec une rétrécissement de la colonne d'air, musculature du cou «remontée». Bouche

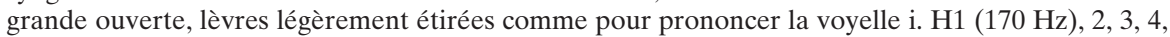
5,6 du bourdon très marqués. Mélodie d'harmoniques H8 $(1360 \mathrm{~Hz}), 9,10(1700 \mathrm{~Hz})$. 


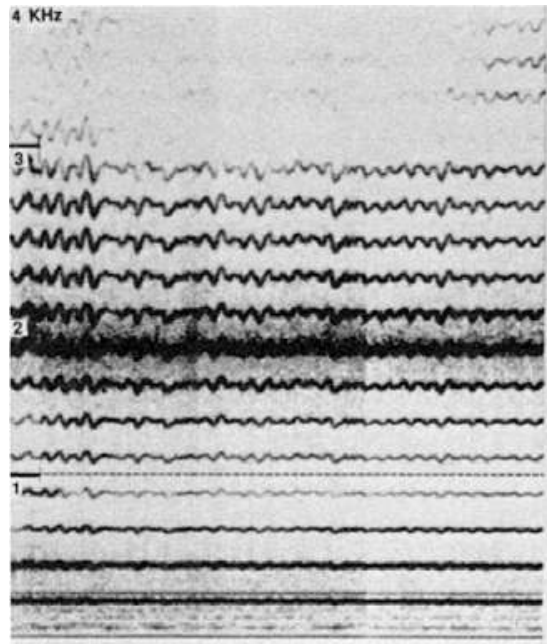

Fig. 35: Essai par Trân Quang Hai.

H1 $(220 \mathrm{~Hz})$; mélodie d'harmoniques H8. Forte contraction abdominale et pharyngienne. Conduit d'air très reserré. La vibration est ressentie plus fortement au-dessus de la pomme d'Adam.
$4 \mathrm{KHz}$

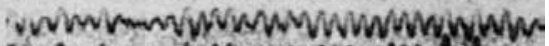

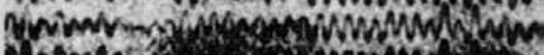

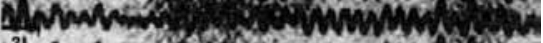

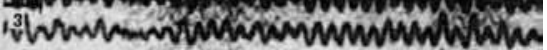

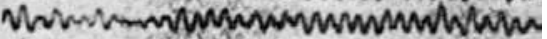

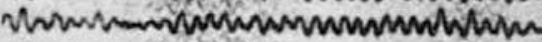

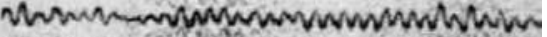

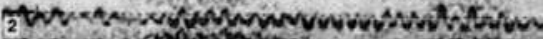

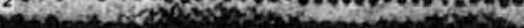

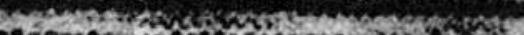

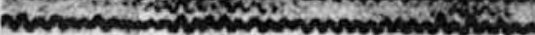

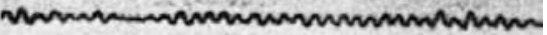

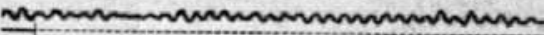

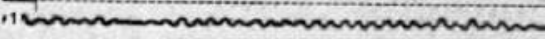

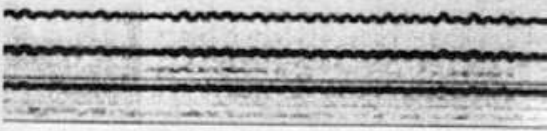

Fig. 36: Essai par Trân Quang Hai.

H1 $(220 \mathrm{~Hz})$; mélodie d'harmoniques H8.

Forte contraction abdominale et pharyngienne. Conduit d'air plus large. La vibration est ressentie plus fortement au-dessous de la pomme d'Adam.

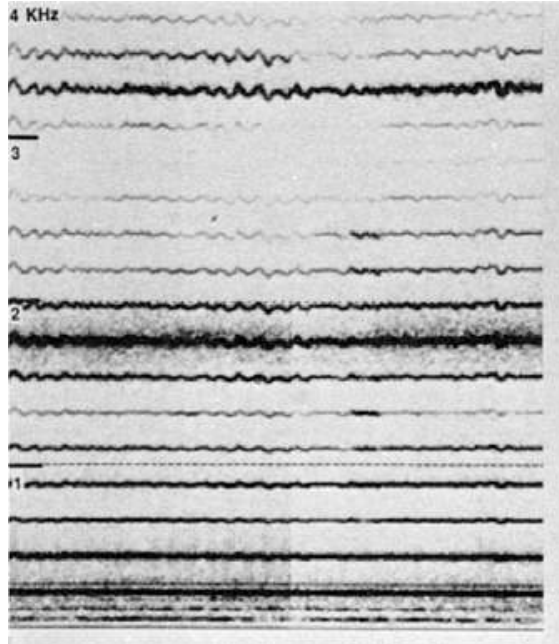

Fig. 37: Essai par Trân Quang Hai.

H1 $(220 \mathrm{~Hz})$; mélodie d'harmoniques H8.

Relaxation abdominale et pharyngienne. Intensité faible; pour obtenir un tracé noir, il fallait compenser en augmentant le niveau d'entrée du Sona-Graph.

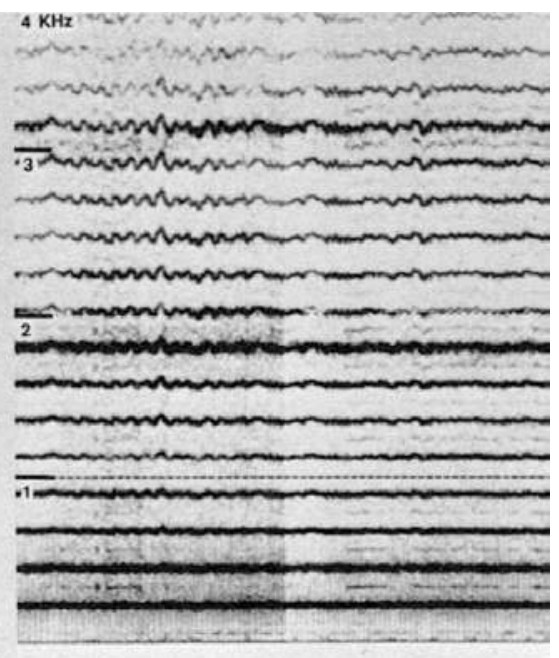

Fig. 38: Essai par Trân Quang Hai.

H1 $(220 \mathrm{~Hz})$; mélodie d'harmoniques H8.

Bouche fermée. Contractions comme pour la fig. 36. Intensité faible; pour obtenir un tracé noir, il fallait compenser en augmentant le niveau d'entrée du Sona-Graph. 


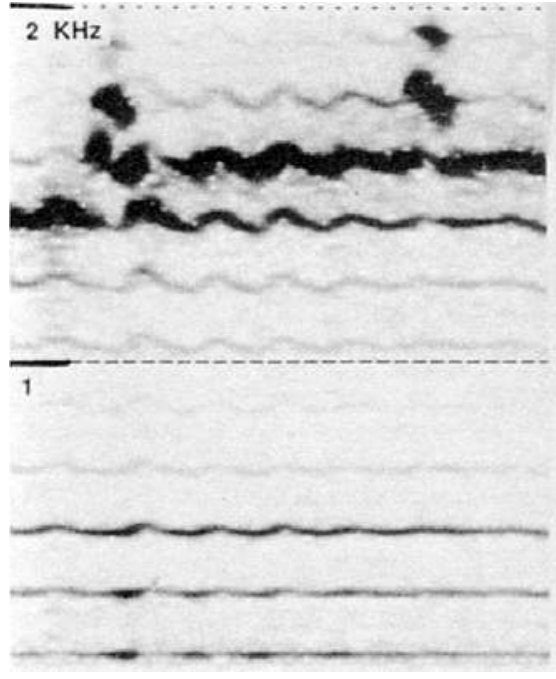

Fig. 39: Sigit tuva (cf. fig. 23). Ornements rythmiques «en ponctuations » sur H10, combinés avec du vibrato.

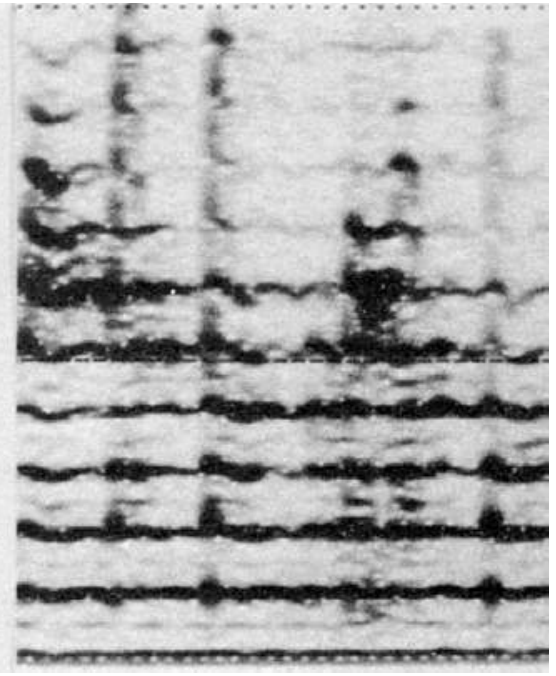

Fig. 40: Chant du Rajasthan (cf. fig. 27). Accents rythmiques marqués par des lignes verticales sur toute l'étendue du spectre.

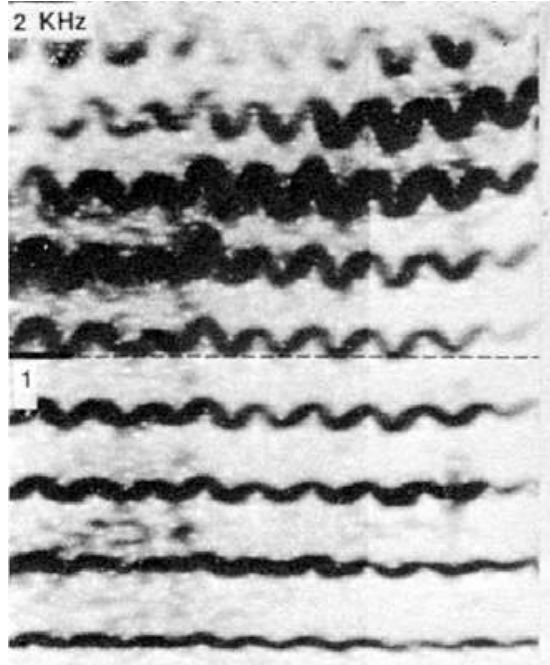

Fig. 41: «Khöömii de ventre» mongol (cf. fig. 25). Vibrato «en ondulations».

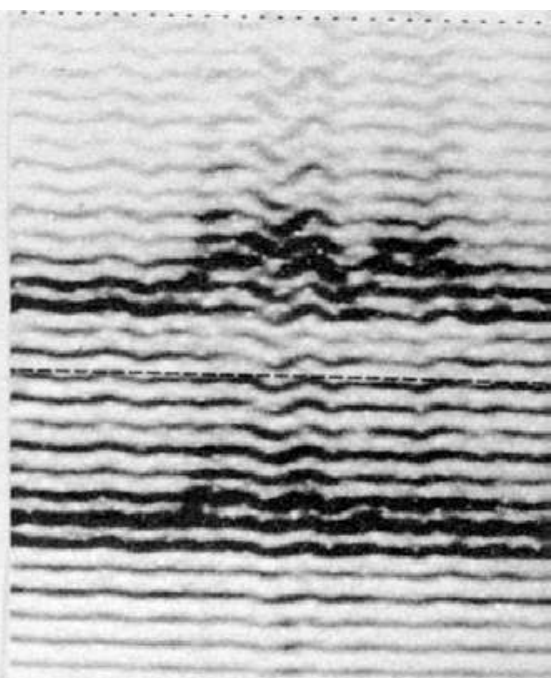

Fig. 42: Kargiraa tuva (cf. fig. 5). Vibrato «en ondulations». 


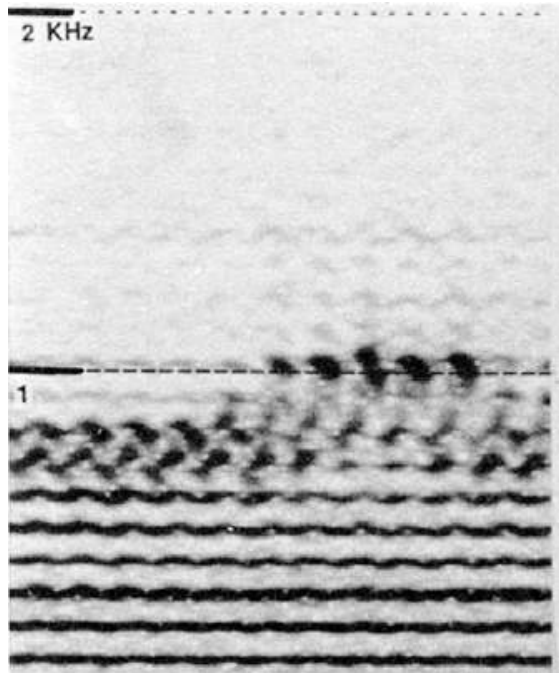

Fig. 43: Borbannadir tuva à fondamental grave (cf. fig. 13). Pulsation «en zigzag» sur la mélodie d'harmoniques à bande large.

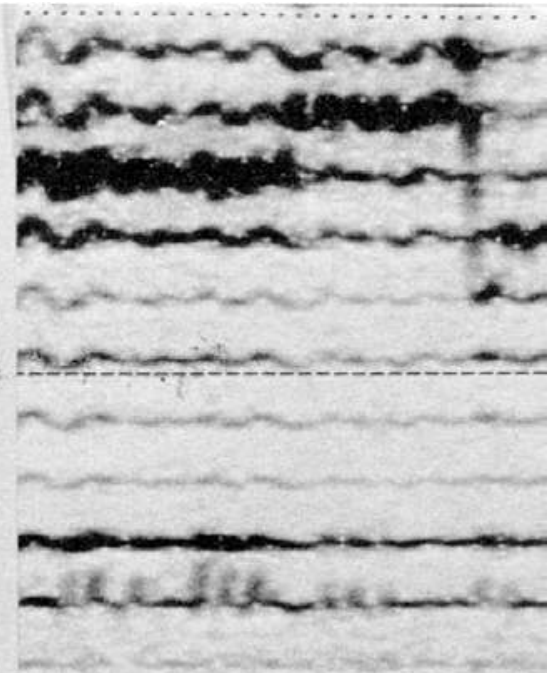

Fig. 44: Borbannadir tuva à fondamental aigu (cf. fig. 22). Accents rythmiques «en hachures» sur H2 par frappement de doigt(s) sur les lèvres.

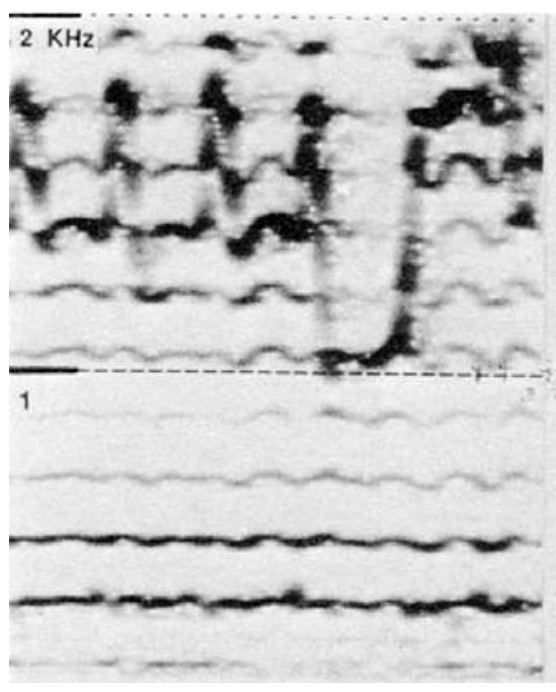

Fig. 45: Khomei tuva (cf. fig. 18). Pulsation rythmique marquée par des traits verticaux alternativement au-dessus et au-dessous de la ligne horizontale.

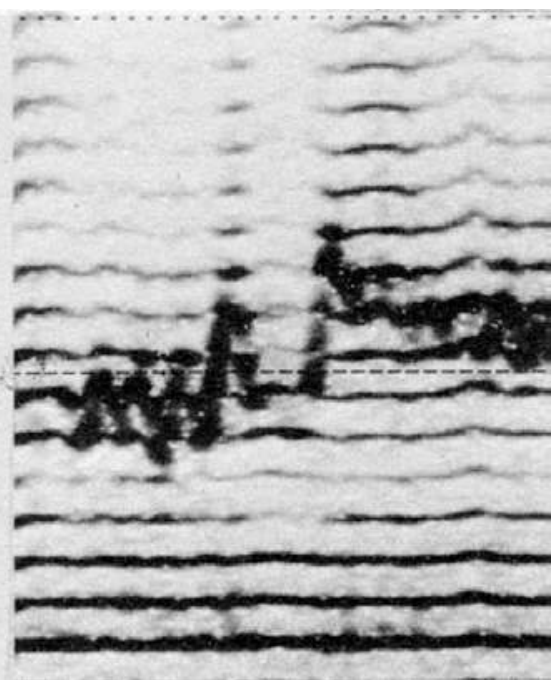

Fig. 46: Ezengileer tuva (cf. fig. 21). Accents rythmiques marqués par des traits verticaux alternativement au-dessus et au-dessous de la ligne horizontale. 


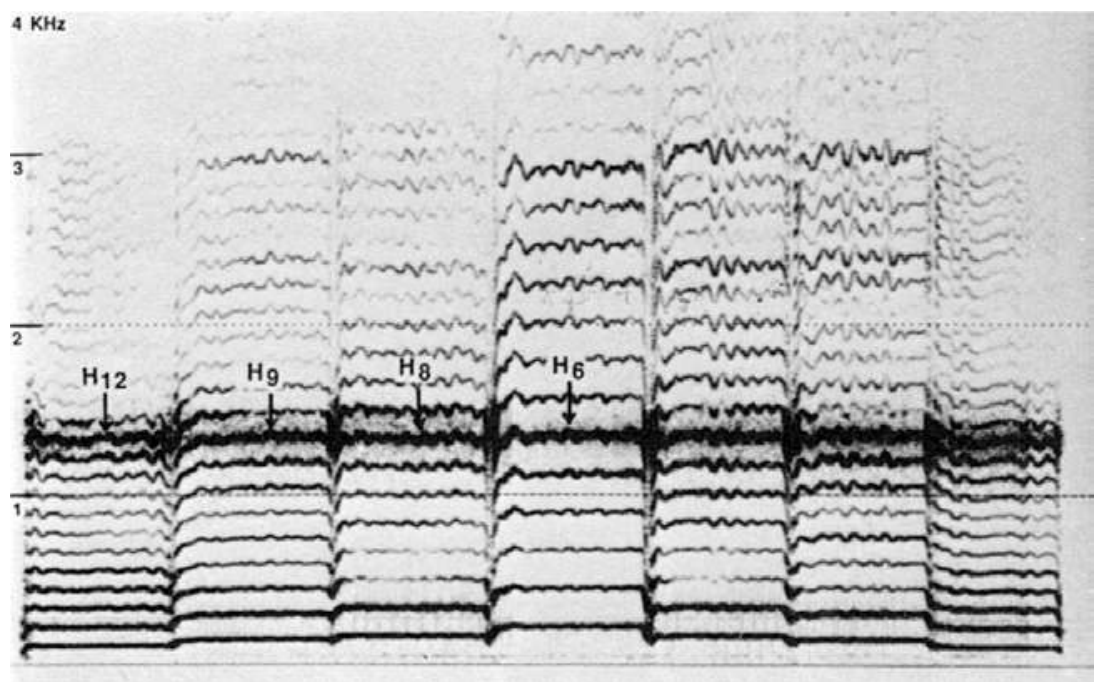

Fig. 47: Expérimentation d'un «bourdon harmonique» dans l'aigu, avec variation du fondamental, par Trân Quang Hai.

Technique à deux cavités. Voix fortement nasalisée. Contraction pharyngienne et abdominale. Échelle montante des fondamentaux La1 $(135 \mathrm{~Hz}), \mathrm{Re} 2, \mathrm{Mi} 2, \mathrm{La} 2(270 \mathrm{~Hz})$, et redescendante au La1. «Bourdon harmonique» de $1380 \mathrm{~Hz}$ réalisé successivement par H12, 9, 8, 6, 8, 9, 12.

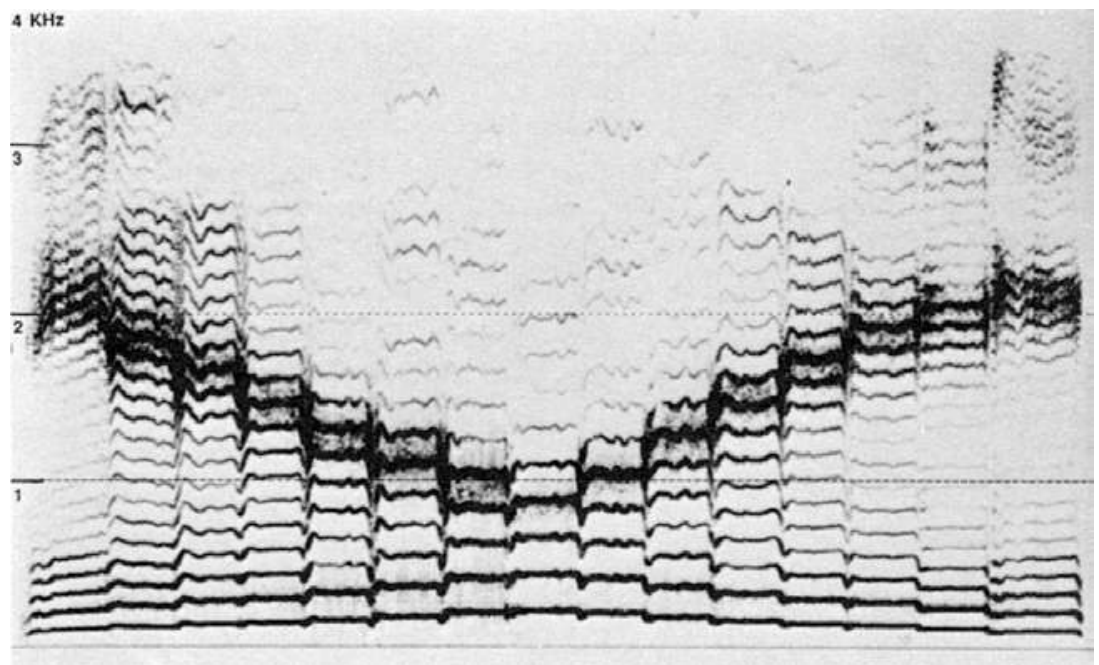

Fig. 48: Expérimentation d'un mouvement contraire entre bourdon et mélodie d'harmoniques, par Trân Quang Hai.

Technique à deux cavités. Voix fortement nasalisée. Contraction pharyngienne et abdominale. Échelle diatonique ascendante des fondamentaux du La1 $(110 \mathrm{~Hz})$ au La2 $(220 \mathrm{~Hz})$ et redescendante. Échelles d'harmoniques en mouvement contraire, descendante de $\mathrm{H} 19$ au H4 et remontante à H19. 